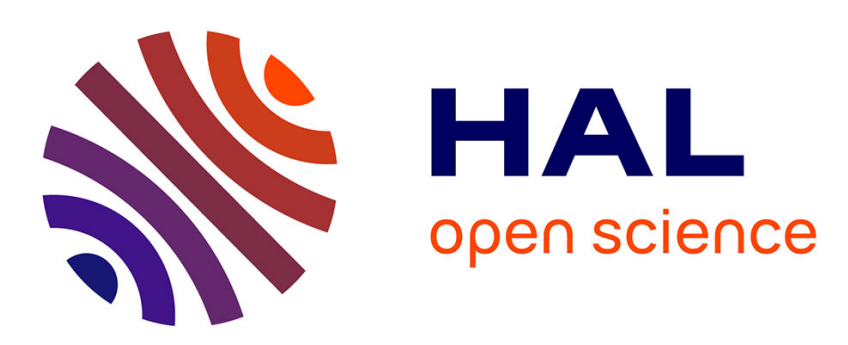

\title{
Surface water maps de-noising and missing-data filling using determinist spatial filters based on several a priori information
}

Filipe Aires

\section{- To cite this version:}

Filipe Aires. Surface water maps de-noising and missing-data filling using determinist spatial filters based on several a priori information. Remote Sensing of Environment, 2020, 10.1016/j.rse.2019.111481 . hal-02377012

\section{HAL Id: hal-02377012 \\ https://hal.science/hal-02377012}

Submitted on 22 Nov 2019

HAL is a multi-disciplinary open access archive for the deposit and dissemination of scientific research documents, whether they are published or not. The documents may come from teaching and research institutions in France or abroad, or from public or private research centers.
L'archive ouverte pluridisciplinaire HAL, est destinée au dépôt et à la diffusion de documents scientifiques de niveau recherche, publiés ou non, émanant des établissements d'enseignement et de recherche français ou étrangers, des laboratoires publics ou privés. 


\title{
Surface water maps de-noising and missing-data filling using determinist spatial filters based on several a priori information
}

\author{
Filipe Aires ${ }^{\mathrm{a}, *}$ \\ ${ }^{a}$ Estellus SAS, Paris, France
}

\begin{abstract}
Satellite observations are used to detect surface waters but uncertainties such as instrument noise or retrieval errors can introduce noise or missing-data in the resulting water maps, especially for datasets at the global scale. In this study, spatial filters based on several a priori information are proposed to reduce noise and perform spatial interpolation to fill missing-data in satellite-based surface water maps such as wetlands, rivers, lakes. Four main sources of a priori of information are considered: (1) historical information at the pixel level, (2) neighbouring information constraints based on a historical record, (3) constraints based on topography, and (4) hydrological constraints based on a floodability index. Experiments are conducted over synthetic but realistic data, as well as over real Sentinel 1 (SAR) and 2 (visible) water map retrievals. Mis-classification quantitative results over these three types of data show that simple determinist spatial filters allow reducing noise and filling missingdata. The four sources of a priori information can be exploited and combined to improve observed water maps. This opens some ways to develop post-processing tools for improving surface water maps at high spatial resolution from missions such as SWOT (Surface Water and Ocean Topography) to be launched in 2020.
\end{abstract}

Keywords: A priori information for surface water, Determinist filters for de-noising and missing-data, neighbourhood constraints, floodability index, topography information 2010 MSC: 00-01, 99-00

\footnotetext{
*Corresponding author

Email address: filipe.aires@estellus.fr (Filipe Aires)

URL: https://vm-wordpress-lerma01.obspm.fr/faires/ (Filipe Aires)
} 


\section{Introduction}

Global distribution and dynamics of surface waters at medium resolution (around $100 \mathrm{~m}$ ) are necessary to satisfy the needs of hydrologists, water and disaster managers, or climate scientists. Permanently and temporarily inundated areas including lakes, rivers, and wet-

5 lands, are important because of their interaction with climate, ecology, and human wellbeing. For instance, nearly 30\% of global methane emissions (Bousquet et al., 2006) originate from wetland areas, risk management responds to inundation patterns (Winsemius et al., 2015), and food security, and rice paddy cultivation relies, in certain regions of the world, on surface waters. In return, surface water ecosystems are affected by human activity, land use, hydrologic alterations, and climate change. Although the global, long-term, frequent, and high-resolution characterisation of all surface water types is beyond the capabilities of current satellite observations (Aires et al. 2018), several types of datasets are being used to document them as well as possible.

Despite the limitations from vegetation canopy and cloud cover (about $70 \%$ of Earth surface at any time) (Wilson and Jetz, 2016), visible satellite observations are a primary candidate for the detection of surface waters from space. Moderate Resolution Imaging Spectro-radiometer (MODIS) observations have been used to derive global surface water products every two days (http://oas.gsfc.nasa.gov/floodmap/). Several datasets have also been built from Landsat imagery: Feng et al. (2014) proposes a global, high-resolution (30 m) inland water body dataset for year 2000, Mueller et al. (2016) focused on the Australian continent by processing a 27-year time series of Landsat imagery, Tulbure et al. (2016) created a three decade dataset (1986-2011) of surface water and flooding over the over the MurrayDarling semi-arid basin, and Verpoorter et al. (2014) mapped an inventory of global lakes: the GLObal WAter BOdies database (GLOWABO) comprises all lakes greater than $0.002 \mathrm{~km}^{2}$ (about 117 million lakes, with a combined surface area of about $5.106 \mathrm{~km}^{2}$ ). Yamazaki et al. (2015) introduced the Global 3 arc-second Water Body Map (G3WBM) based on Landsat imagery. Pekel et al. (2016a) recently produced a new Global Surface Water Explorer (GSWE) dataset also from Landsat imagery but using the full 32-year record, allowing for a better description of the trends of surface waters and their occurrence. The limitations for the use of visible/infrared (VIS/IR) instruments are quite straightforward: these waves do not penetrate clouds nor vegetation. As a result, VIS/IR retrieved inundation 
maps can have missing or corrupted data. For instance, Sentinel-2 data has a large amount of missing-data from the presence of clouds in the tropics. These missing/erroneous data can cover large areas (difficult to interpolate them spatially) or can be present at the pixel level, so techniques to interpolated missing areas or to reduce noise on the maps are necessary.

Synthetic Aperture Radar (SAR) data have the potential to retrieve surface waters as demonstrated for instance by Santoro et al. (2010) using ENVISAT-ASAR, or more recently using the Sentinel 1 mission (Pham-Duc et al., 2017). This surface water retrieval can be done at a resolution of up to $10 \mathrm{~m}$ (Cao et al., 2019). SAR data can also be used to capture sub-canopy inundation (L-band) (Plank et al., 2017). Although existing SAR retrievals from a number of sensors cover a large extent of the globe, their use for mapping surface inundation has been protracted due to the local calibration needed for accuracy. The past or current availability of the data has not yet allowed for the production a full global highspatial resolution surface water dataset from SAR data, although such initiatives have been suggested in the past, e.g. Westerhoff et al. (2013). This results from the fact that the necessary algorithms have limitations in terms of robustness, accuracy and automation. For instance Shen et al. (2019) comment on the fact that automation and robustness have not been achieved yet for vegetated areas when using L-band observations. This means that misclassifications and missing data will be present in this type of retrievals. They attribute errors to three different sources:

- Water-like surfaces: smooth surfaces (at the scale of the measuring wavelength) even bare soil or shadowed areas have scattering properties similar to those of water surfaces. These ambiguities can introduce erroneous water pixels (i.e. over-detection). For instance, Giustarini et al. (2013) and Matgen et al. use a change-detection algorithm to limit these over-detections.

- Noise-like speckle: This is one major disadvantage of SAR over optical images. This is not a real noise (see Lee and Pottier (2009) for a definition) but homogeneous and continuous areas can exhibit strong inhomogeneities in SAR images due to this speckle. It increases with the spatial resolution. Filtering techniques have been developed for that purpose, at a price of the degradation of the spatial resolution of the image.

- and geometric correction: Due to the limited accuracy of input elevation data and 
orbit accuracy, it is frequent to see location errors at the level of a few pixels.

So far, algorithms have only partially addressed these issues and human intervention to reduce our-detection as well as filtering to reduce under-detection are needed (Shen et al., 2019). Therefore, techniques to interpolated missing areas and to reduce noise on retrieved maps need to be used as a post-processing step to improve quality of retrieval results. For instance, Pulvirenti et al. (2011a) have developed an image segmentation methods based on a dilution and an erosion steps to remove isolated groups of water pixels and small holes in water bodies) to reduce speckle impact. Solutions could come from the use of auxiliary information such as land cover, elevation, and so on (see Pulvirenti et al. (2011b)). This is the option tested here in this paper.

The GIEMS-D3 (Global Inundation Extent from Multi-Satellites) is a global water extent dataset at the 3 arc-second $(\sim 90 \mathrm{~m})$ spatial resolution, see Aires et al. (2017). GIEMS-D3 is based on the coarse resolution GIEMS database from a retrieval scheme that combines satellite observations in the visible, near-infrared, and passive/active microwaves (Prigent et al., 2007, 2012; Papa et al., 2010) over a 15-year period (1993-2007). The advantage of this dataset is that it provides surface wetland estimates even below the vegetation Aires et al. (2018).

There is clearly a need to invest more time in retrieval algorithms and potentially perform data fusion in order to obtain a global, long-term, reliable, and high-resolution dataset of water extent from these several type of observations. As discussed earlier, all these satellite datasets can suffer from retrieval errors (e.g. instrumental noise, retrieval errors, or erroneous/missing auxiliary information). The retrieved surface water maps can also have missing-data, due to the presence of clouds or vegetation, or due to retrieval difficulties. Furthermore, all these surface water satellite retrievals can be improved by combining them with other independent auxiliary information. The goal of this paper is first to present several sources of a priori information that are pertinent constraints for the water presence. Four sources of a priori information will be considered: (1) Historical information at the pixel level, (2) Neighbouring information constrains based on historical information, (3) Constraints based on topography information, (4) Constraints based on hydrology information. It will be investigated here if these four sources of information can help post-process corrupted surface water maps by reducing noise in retrieval and fill missing-data. 
Several determinist filters are then introduced and tested to exploit these sources of $a$ priori information to de-noise maps or fill missing-data. These new methods are designed to process surface water maps retrieved from satellite observations (whatever their origin), and not the satellite measurement itself such as the backscatter signal from a SAR instrument (Deledalle et al., 2014). The a priori information that are proposed here (historical record, neighbourhood constrains, elevation, or floodability index) are general and not specific to a particular type of observations. However, they could be optimised to become more specific to a particular type of information with some specificities in terms of uncertainties (i.e. instrument noise, wrong assumptions, limited information, or retrieval errors) or in terms of missing-data (presence of clouds, instrument recalibration, etc.).

Section 2 presents the datasets used in this study. The determinist filters are introduced in Section 3. Results are presented in Section 4 for the de-noising and in Section 5 for the filling of missing-data. A discussion is provided in Section 6. Finally, conclusions and perspectives are provided in Section 7 .

\section{Datasets used in this study}

\subsection{Sentinel observations}

Sentinel data have been gathered and processed over the Mekong in Vietnam and Camargue in south of France. Clouds are predominant over the Mekong region (Aires et al., 2018) so Sentinel 1 SAR retrievals are considered for this region. Cloudiness is less of a problem over Camargue so Sentinel 2 Visible retrievals are considered instead. The spatial resolution of Sentinel data is $30 \times 30 \mathrm{~m}$, both for the SAR and the visible data.

\subsubsection{Sentinel 1 SAR observations over Mekong}

Two Sentinel 1 SAR images are available over the Mekong: Instantaneous and Reference, both during 2016. Fig. 1 represents the Instantaneous (Fig. 1.A) and the Reference (Fig. 1.B) images. The Instantaneous images are of lesser quality, for they contain instrument and retrieval noises. The Reference image is the averaging over a 40-day period preceding the Instantaneous image. It is considered to have less noise due to this time-averaging.

In a classification problem, four diagnostics are generally defined: (1) The False Positives (FP) where the sample is erroneously classified as positive (i.e. inundated here); (2) the False Negatives (FN) where the sample is erroneously classified as negative; (3) the True Positives 
(A) Instantaneous

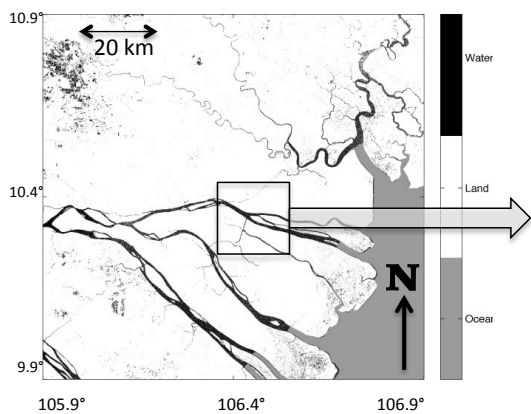

(D) Reference

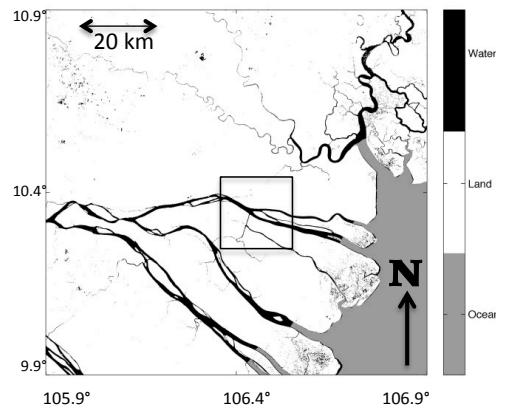

(B) Inst. zoom

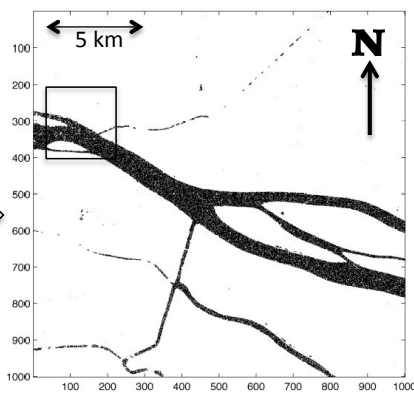

(E) Ref. zoom

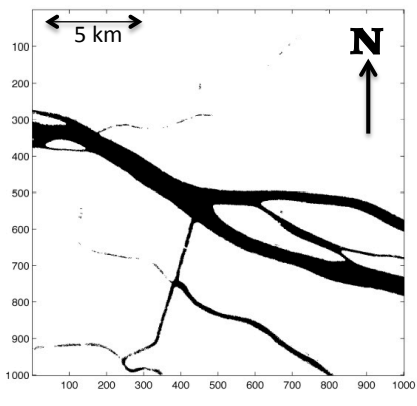

(C) False Positive (297,099 pixels)

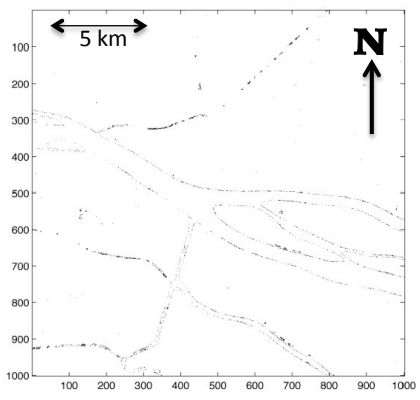

(F) False Negative $(418,457$ pixels)

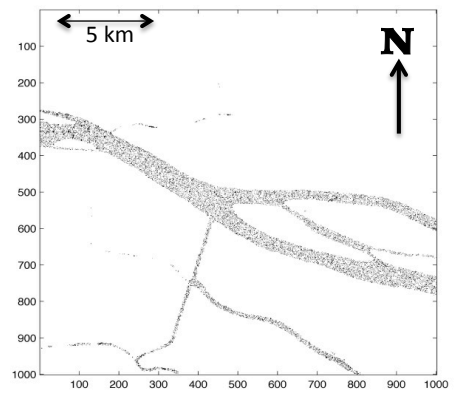

Figure 1: Mekong region. First column: SAR data from Sentinel 1 for the Instantaneous (A) and Reference (B) images. Second column is identical with a zoom on one smaller region (B and E). Third column is the "false" positive (C) pixels (i.e. inundated pixels in Instantaneous but not in Reference images), followed by the "false" negative (F) pixels (i.e. dry pixels in Instantaneous but not in Reference images). The spatial domain of sub-labels (B, C, E and F) is represented by the black square in (A) and (B). Sub-domain in a black square in (B) will be used later on (in Fig. 9).

(TP) where the sample is correctly classified as positive; and (3) the True Negatives (TN) where the sample is correctly classified as negative. These diagnostics can be provided in absolute number of samples, or they can be given in percentage. The third column in Fig. 1 represents the FP (C) and FN (F) pixels of the Instantaneous image when considering the Reference as the truth (which is a simplification).

Among the 30,140,100 pixels of the Instantaneous image, $10.5 \%$ are ocean, and $89.5 \%$ are land. The de-noising and spatial interpolation methods will consider only the 26,989,240 land pixels (the "work space" in the following).

Tab. 1 provides the confusion matrix between the Instantaneous and Reference images. It can be noted that most negative values in the Instantaneous image are TN values 
$(\mathrm{TN}=98.36 \%)$ but many $\mathrm{FP}$ errors are present in the positive values $(\mathrm{FP}=20.20 \%)$. There are more FN pixels $(418,457)$ than FP ones $(297,099)$, but there are much less positive pixels so the percentage is higher. Fig. 1 shows the spatial distribution of these differences: FP pixels are located in the edge of the rivers $(\mathrm{C})$, and $\mathrm{FN}$ pixels are in the center of the river (F). The total number of erroneous pixels is $2.37 \%$ of the total image, it represents $2.65 \%$ of the work space (i.e. non-oceanic).

\begin{tabular}{|r|rr|}
\hline \multicolumn{3}{|c|}{ Mekong } \\
\hline Inst. & $\mathrm{N}=25,518,940$ & $\mathrm{P}=1,470,300$ \\
$\mathrm{~N}=25,397,582$ & $\mathrm{TN}=25,100,483$ & $\mathrm{FP}=297,099$ \\
$\mathrm{P}=1,591,658$ & $\mathrm{FN}=418,457$ & $\mathrm{TP}=1,173,201$ \\
& $1.64 \%$ & $79.80 \%$ \\
\hline Errors=715,556 & $2.65 \% /$ work & $2.37 \% /$ total \\
\hline & $\mathrm{Camargue}$ & \\
\hline Inst. & $\mathrm{N}=6,564,010$ & $\mathrm{P}=921,875$ \\
\hline $\mathrm{N}=6,503,959$ & $\mathrm{TN}=6,480,989$ & $\mathrm{FP}=22,970$ \\
& $98.74 \%$ & $2.49 \%$ \\
\hline $\mathrm{P}=981,926$ & $\mathrm{FN}=83,021$ & $\mathrm{TP}=898,905$ \\
& $1.26 \%$ & $97.51 \%$ \\
\hline Errors=105,991 & $1.42 \% /$ work & $0.35 \% /$ total \\
\hline
\end{tabular}

Table 1: Confusion matrix for the Instantaneous and Reference images over the Mekong (Top) and the Camargue (Bottom), see Figs. 1 and 2, $\mathrm{N}$ is for Negative and $\mathrm{P}$ for Positive pixels. "Work" is for the working space (i.e. pixels that are not permanently wet or dry), they can be either wet or dry pixels. Pixels over the ocean are permanently wet so they are not part of the "work" space. "Total" is for all the pixels of the image.

\subsubsection{Sentinel 2 visible observations data over Camargue}

Two images are again represented in Fig. 2; the Instantaneous (A) and Reference(B) images over the Camargue region, but from the Visible/Sentinel 2 instrument. A cloud mask $(\mathrm{C})$ is also represented for the Instantaneous image, it indicates the missing parts 


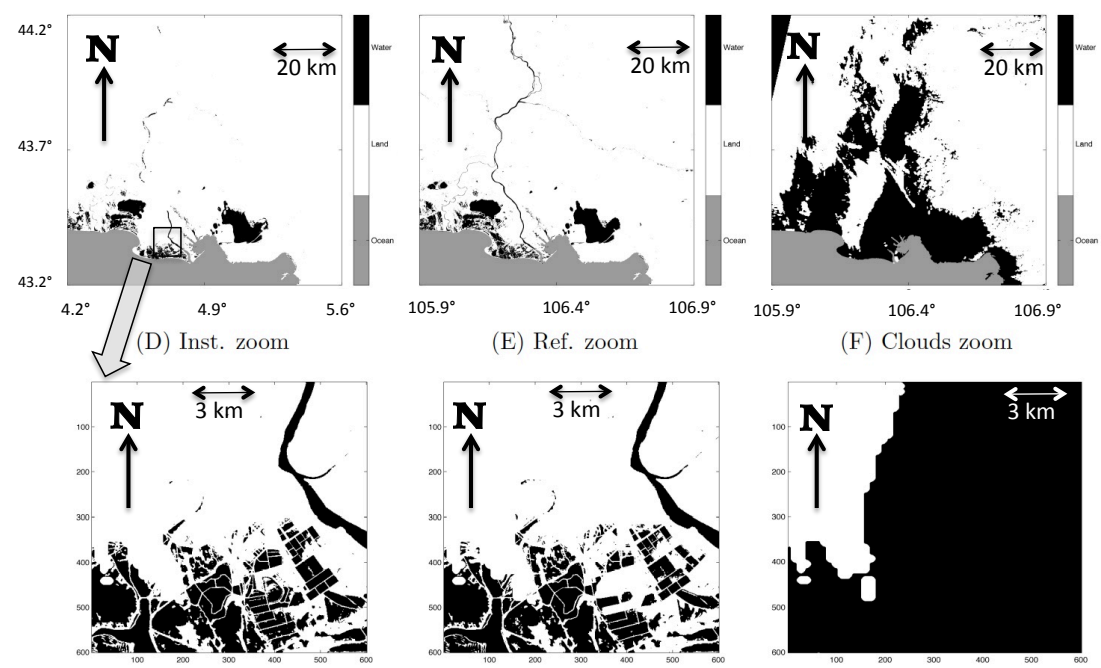

(G) False Positive

(H) False Negative
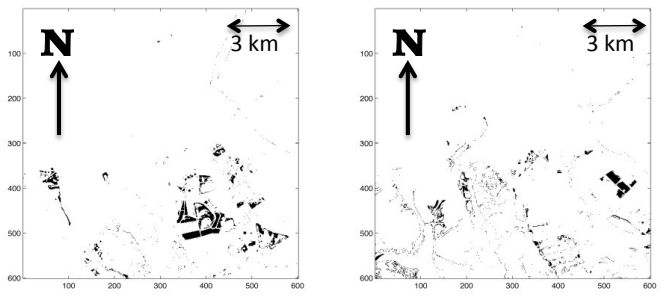

Figure 2: Camargue region. First row: Visible data from Sentinel 2, from left to right: Instantaneous (A), Reference (B), and Clouds (C). Second row (D,E,F): Same as first line, but zoomed over one region represented by the black square in (A) (see Fig. 12). Third row: FP (G) and FN (H) pixels (with Reference as truth, see text) over the (A) square too.

of the image. The FP $(\mathrm{G})$ and FN $(\mathrm{H})$ images are also represented. No noisy pixels are observed so this visible Sentinel 2 image will not be used for the de-noising experiences. It will be used instead for the missing-data filling experiments.

\subsection{GIEMS-D3 dataset}

A multi-sensor technique has been developed to estimate surface water extent at global scale (Prigent et al., 2007, 2012; Papa et al., 2010). The method exploits the complementary sensitivities of different satellite observations to surface characteristics (e.g., water, vegetation, soil). The following satellite observations were used to generate GIEMS: passive microwaves, active microwave, and visible and near-infrared reflectances (Prigent et al., 2001). Note that GIEMS estimates include all surface waters such as rivers, floodplains or lakes indiscriminately. GIEMS is available at (http://lerma.obspm.fr/spip.php?article91lang=en). 
Downscaling methods have recently been developed to improve the spatial resolution of

155 „GIEMS estimates from $25 \mathrm{~km}$ to $500 \mathrm{~m}$ (15 arc-second, GIEMS-D15) (Fluet-Chouinard et al., 2015) (http://www.estellus.fr/index.php?static13/giems-d15) and $90 \mathrm{~m} \mathrm{(3} \mathrm{arc-second,}$ GIEMS-D3) (Aires et al., 2017). GIEMS-D3 has been assessed by analysing its spatial and temporal variability, and evaluated by comparisons to other independent satellite observations (Aires et al., 2017). GIEMS-D3 has also been compared to other global high spatial resolution datasets and it was shown in Aires et al. (2018) that this is a good source of information, especially for vegetated inundated areas not captured by other datasets.

\subsection{Global Surface Water Occurence (GSWO) a priori from Landsat}

An important source of information for de-noising or fill the missing-data in surface water maps would be a historical record of the same surface water maps, from the same instruments. It is also possible to use a historical record from another instrument if both instruments have nearly identical sensor characteristics (e.g. for optical sensors the bandwidths) so that their data can be used interchangeably. In this way for instance both instruments are impacted in the same way by the presence of vegetation. The GSWO dataset (Pekel et al., 2016a) uses three million Landsat satellite images to quantify inundation over 32 years (from 1984 to 2015) at a $30 \mathrm{~m}$ spatial resolution. The GSWO dataset is freely available at: https://globalsurface-water.appspot.com/. Each Landsat pixel has been classified as open water, land, or non-valid observation using an expert system. Open water is defined as any feature of water larger than $30 \mathrm{~m} \times 30 \mathrm{~m}$ open to the sky, including fresh and saltwater. No seasonal information is available, the occurence has been computed over the whole record, regardless of the season. This aspect will be investigated in the future, if this is possible from the GSWO facility. GSWO database (Pekel et al., 2016b) provides the probabilities of water occurence for each pixel based on a Landsat record of 32 years. Note that since these observations are on the visible range, this database is reliable for open water but is less appropriate for highly-cloudy regions or vegetated areas as shown in Aires et al. (2018).

\subsection{MERIT topography a priori}

This a priori information is not based on surface water maps itself, so it is totally different in nature than the preceding a priori information. However, it is evident hydrologically that surface waters are strongly constrained by topography. It has been shown that topography is a reliable source of information for determining the presence of surface waters, see 
Fluet-Chouinard et al. (2015) and Aires et al. (2017). This information is not pertinent everywhere in the world because inundation can be controlled by human activity instead of topography. But very locally, lower elevation in close pixels generally means higher chance of inundation. The "Multi-Error-Removed Improved-Terrain" (MERIT) DEM is used here; it is freely available at http://hydro.iis.u-tokyo.ac.jp/ yamadai/MERIT_DEM/ see Yamazaki et al. (2017).

\subsection{Floodability index a priori}

In Aires et al. (2017), a floodability index model was defined to estimate locally a proxy of the probability to be flooded for each pixel $i$ from topography information. The topographic attributes $X$ that were used are: Elevation over nearest river, slope, distance to nearest river, and flow accumulation. The conditional probability $P[I(i)=1 \mid X=x]$ of pixel $i$ to be inundated knowing topography $w$, as modelled using an artificial Neural Network (NN) with the vector of topographic variables $x$ as inputs, and the inundation probability estimate as output.

Fig. 3 represents, over the Mekong and the Camargue regions, the available a priori information that will be used in this study.

\section{Spatial filters for de-noising and filling missing-data}

\subsection{Filtering problem}

The inundation maps to be de-noised or filled in can be noted as:

$$
O=I+\varepsilon
$$

with $I$ the correct binary image ( 0 or 1 in each one of the $i=1, \cdots, N$ pixels), $O$ the observed image, and $\varepsilon$ the corruption of the data that can originate from an erroneous switching of pixels due to noise or from missing-data. The goal of this study is to obtain an estimate $\mathcal{F}(O)$ as close as possible to the correct image $I$ : The filter $\mathcal{F}$ is asked to de-noise or fill the missing-data in observed image $O$. Random filters (e.g. Markov chains) can be used for this purpose, but they are generally computationally expensive as they request the convergence of an iterative algorithm spanning several times all the pixels. The focus is here instead on deterministic filters. 
(A) GSWO/Mekong

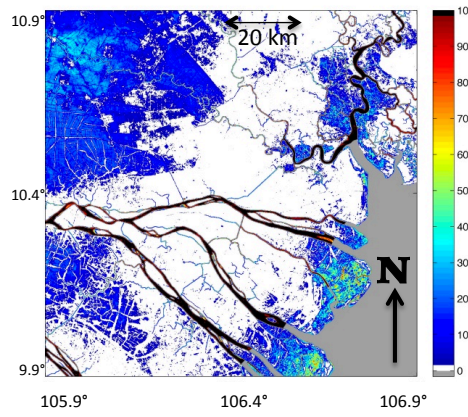

(D) GSWO/Camargue
(B) MERIT DEM/Mekong

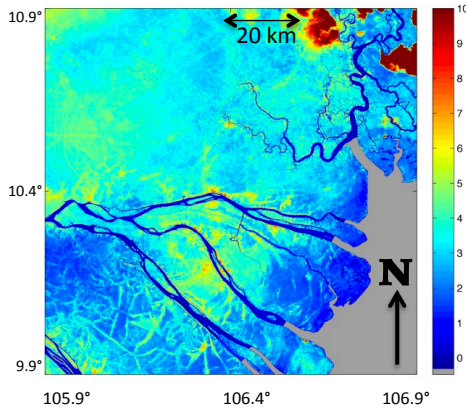

(E) MERIT DEM/Camargue
(C) Floodability Index/Mekong

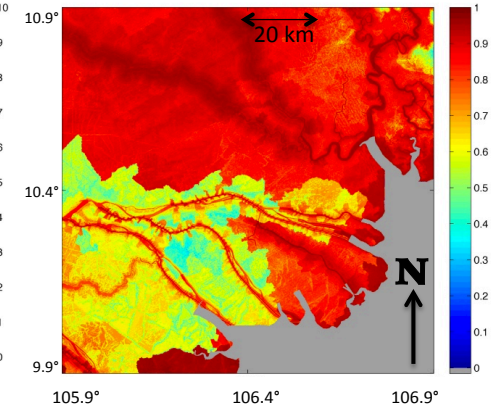

(F) Floodability Index/Camargue
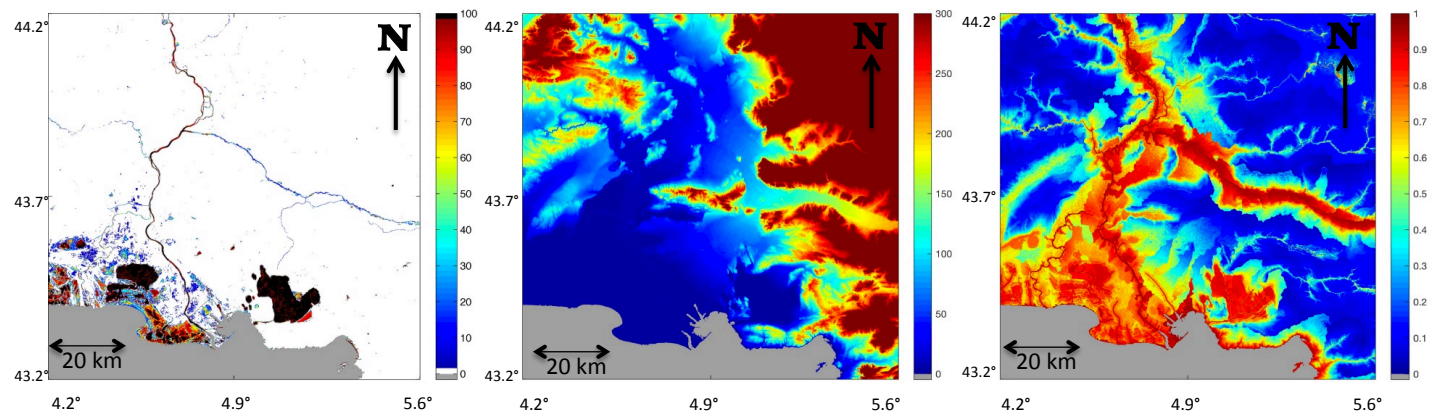

Figure 3: A priori information available over the Mekong (Top) and the Camargue (Bottom) regions, from Left to Right: GSWO, MERIT DEM, and Floodability Index.

It will be investigated here if the a priori information of Section 2 can be exploited to post-process corrupted surface water maps in order to reduce noise, and fill missing-data. This type of methods cannot provide a perfect solution, they even can introduce errors into correct pixels during the de-noising process. Tab. 2 describes the four sources of a priori information used in this study with the corresponding filters characteristics introduced in this section.

The de-noising tries to obtain the right compromise between the observation (i.e. corrupted surface water map) and the a priori information. As a general principle, for the de-noising of the image $O$, the idea is to check in each pixel if there is no big aberration in the observation, and then to correct it. This approach needs to be conservative because it can be dangerous to change too much the original observation $O$. For instance, if $15 \%$ of the pixels are corrupted by noise, and $85 \%$ are good data, applying a filter on each one of the pixels will be good on the $15 \%$ of points, but could corrupt the $85 \%$ remaining good data. 


\begin{tabular}{|l|llll|}
\hline & Origin & Type & Feature & Advantage \\
\hline Filter 1a & Historical record & Prob., all months & Pixel-based & Simplicity \\
Filter 1b & Historical record & Prob., seasonal & Pixel-based & Simplicity \\
\hline Filter 2 & Historical record & Local water & Neighbourhood & Homogeneous or location-specific \\
& & conditions & & External info or not \\
\hline Filter 3 & Topography & Elevation & Neighbourhood & External information \\
\hline Filter 4 & Hydrology & Floodability Index & Neighbourhood & External information \\
\hline
\end{tabular}

Table 2: The four sources of a priori information and their corresponding spatial filters characteristics.

Like often, the right balance needs to be found.

For the missing-data filling, the spatial filters try to identify the best filling solution based on the available a priori information, but the working space (i.e. missing pixels) is well defined in this case.

\subsection{Filter $1 a$ \& b: Pixel-scale probability}

This filter is based on a historical record of the surface water masks. This historical record can be based on a database of observed maps $O$ (from the same instrument), even if the database is corrupted by noise or missing-data; or it can be based on maps from another instrument (such as the GSWO dataset from Landsat, see Section 2.3).

\section{Filter 1}

$$
\begin{aligned}
& I(i)=1 \text { if } \theta_{i} \geq\left(1-\tau_{1}\right) \\
& I(i)=0 \text { if } \theta_{i} \leq \tau_{1}
\end{aligned}
$$

If threshold $\tau_{1}=0$, this filter will characterise which pixels are permanently inundated or dry. This filter has a first application as it can be used to define the pixels that had some variability during the historical record on which it is worth working, and those that had no variability at all and could be omitted from the filtering. If $\tau_{1}=0$, this mask supposes that the historical record is reliable so that newly inundated or dry pixels will not be considered. It directly depends on the quality and representativity of the historical record and the resulting probabilities $\theta_{i}$. If this is not the case, $\tau_{1}$ should be $>0$.

When $\tau_{1}$ is increased, the probability $\theta_{i}$ is used to define the state of pixel $i$ to be inundated or not. Note that symmetric thresholds $\tau_{1}$ and $\left(1-\tau_{1}\right)$ are used for high and small 
probabilities, but this could be optimised independently, for the two cases. The threshold

(1) Homogeneous neighbourhood system for the Sentinel experiments - In this a priori information, a Sentinel image (the Reference) is used in order to infer some neighbourhood constraints. It is not possible here to make historical statistics for each pixel because a time record long enough is not available. Therefore, a single image is used in order to generate a generic statistics applicable to each pixel. Let $V(i) \in[0,8]$ be the number of inundated 
(A)

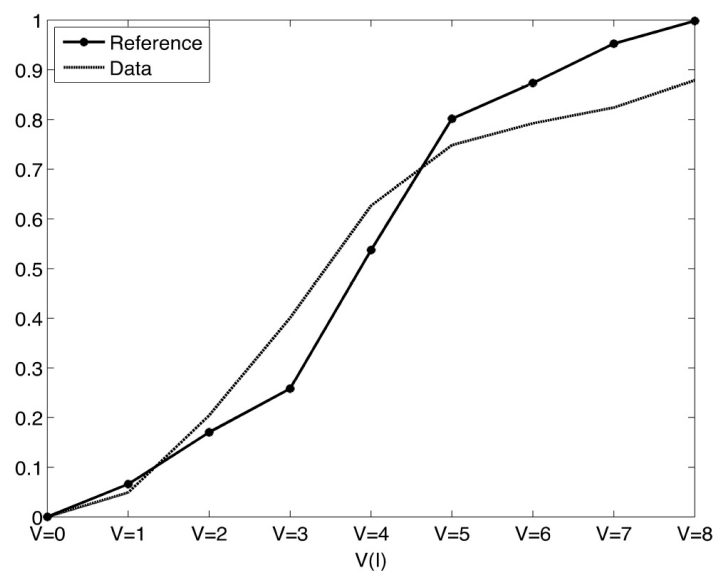

(B)

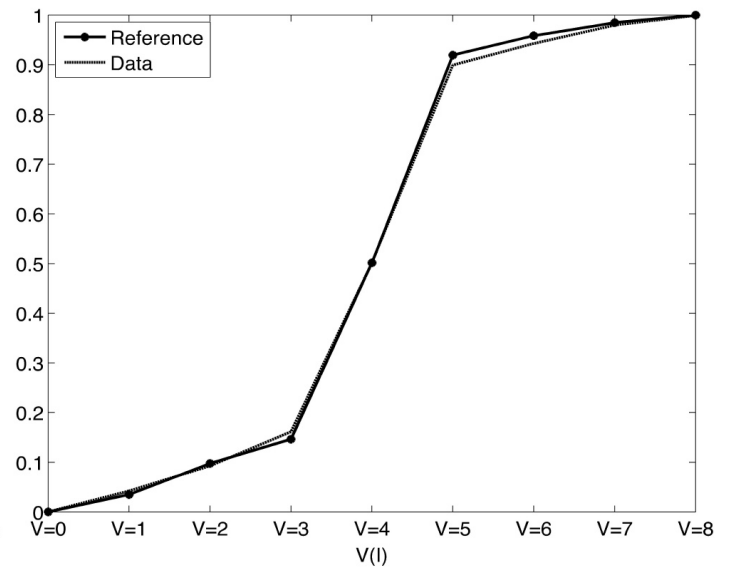

Figure 4: $\operatorname{Prob}(I(i)=1 / V(i)=n)$ for $n=0, \cdots, 8$; for Mekong (A) and Camargue (B). Statistics are performed on the Instantaneous (continuous) and the Reference (dotted) images.

pixels included in the eight pixels $\mathcal{N}(i)$ surrounding pixel $i$. Fig. 4 represents:

$$
\operatorname{Prob}[I(i)=1 \mid V(i)=n], \text { for } n=0, \cdots, 8 .
$$

Note that this conditional probability is valid for $n=0$ too, it just represents the probability of the pixel being inundated when no neighbouring pixel is inundated. This statistics is performed on all the pixels of the Instantaneous and the Reference images for comparison purpose, for the Mekong (left) and the Camargue (right) regions. It can be noted that in the Mekong case, there is a discrepancy between the two sets of probabilities for high $V(i)$ values due to the noise on the Instantaneous image (Fig. 1). This discrepancy almost disappear for the Camargue because there is almost no noise in this case (Fig. 2). The objective of the neighbourhood-based filter will be to get the two sets of probabilities closer, by adjusting aberrant cases in the two extreme cases: when $V(i)$ is small (less important here for the FP cases on the river edges), or large (important here for the FN cases inside the river).

(2) Pixel-based neighbourhood system - Again, a simple, non-oriented neighbourhood system is used by considering $V(i)$, i.e. the number of inundated pixels in the neighbourhood $\mathcal{N}(i)$ of pixel $i$. Instead of proposing a global statistics for all the pixels of the image, these probabilities are estimated for each individual pixel of the image. In order to use this neighbourhood term efficiently, an a priori estimation of all the $P[I(i)=1 \mid(V(i)=n)]$ is 
required for all $n$. However, a configuration $V(i)=n$ might have never occurred in the historical record, or it might have happened so rarely that the estimated probability is not reliable enough. In order to solve this issue, a linear interpolation in $n$ is chosen for all the missing $n$-values. This linear interpolation is performed for each pixel $i$ in order to build a complete a priori dataset on Eq. (2).

Fig. 5 illustrates this interpolation on $n$ for several pixels. Red dots are probabilities of Eq. (2) estimated on the historical record. Black dots represent the linearly interpolated points. The probability function should be increasing with $n$ : the higher the number of neighbouring pixels inundated, the highest the probability for pixel $i$ to be inundated. For extrapolating higher $n$ values, the last available probability is used and supposed to be constant with an increasing $n$.

(A)

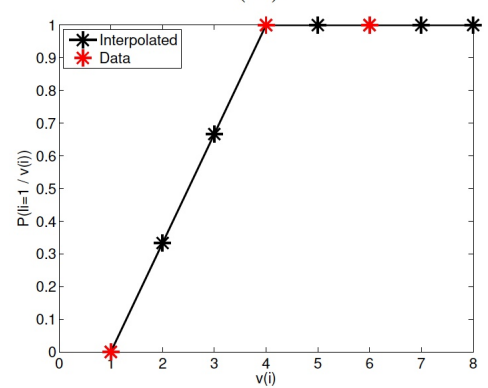

(B)

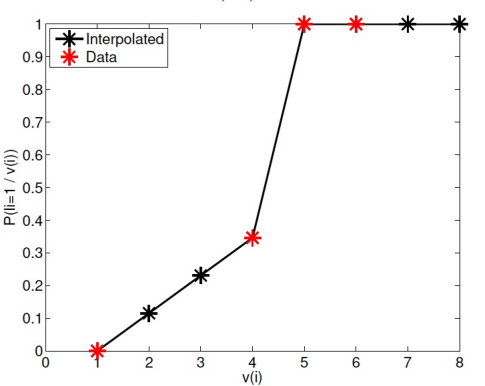

(C)

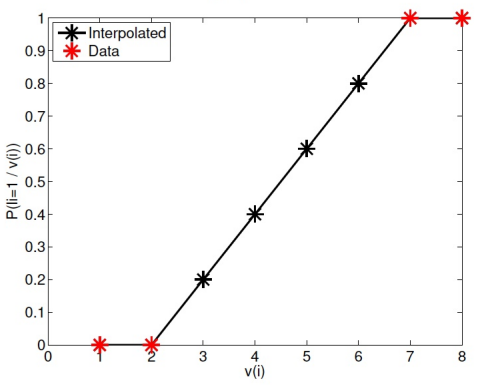

Figure 5: Illustration of the interpolation of $P[I(i)=1 \mid(V(i)=n)]$ (Eq. (2)) for three randomly chosen pixels. Red stars are estimations from the historical data, black stars are the resulting linear interpolation.

Fig. 6 represents the conditional probabilities $P[I(i)=1 \mid(V(i)=n)]$ for increasing $n=$ $0,2,4,6$, and 8 , before (Left) and after (Right) the linear interpolation in $n$. It can be seen in this figure how the $P[I(i)=1 \mid(V(i)=n$ ) (for $n=0$ to 8 ) incomplete statistics have been spatially filled due to the interpolations in Fig. 5. Less values are missing after the interpolation so our a priori became more complete and robust. As expected, the probabilities of being inundated increase when $n$ increases.

It is then possible to use the probability of pixel $i$ to be inundated conditional to its neighbouring inundated pixels $V_{i}(O)$ to correct image $O$. As mentioned earlier, this conditional probability can be pixel-specific (if the historical record was long enough to estimate statistics for each pixel), or it can be an average over the full image. Since no long historical 


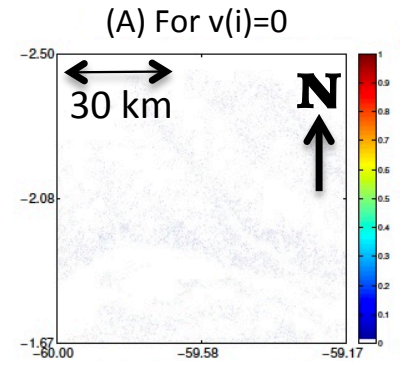

(C) For $v(i)=2$

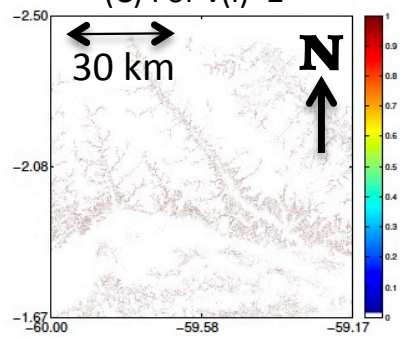

(E) For $v(i)=4$

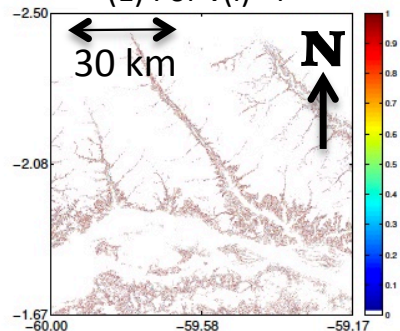

(G) For $v(i)=6$

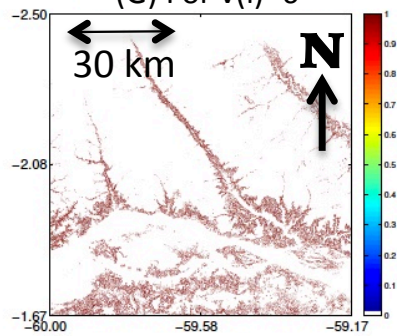

(I) For $v(i)=8$

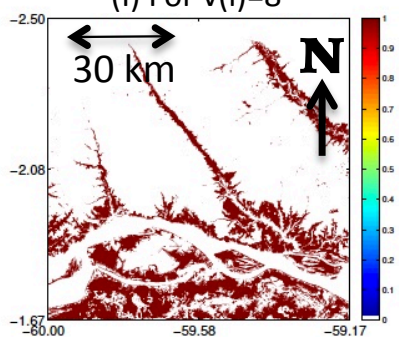

(D) For $v(i)=2$

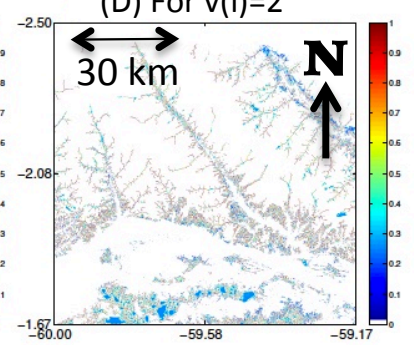

(F) For $v(i)=4$

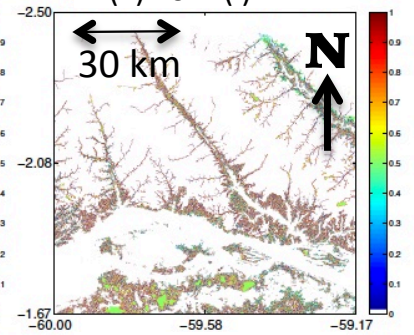

(H) For $v(i)=6$

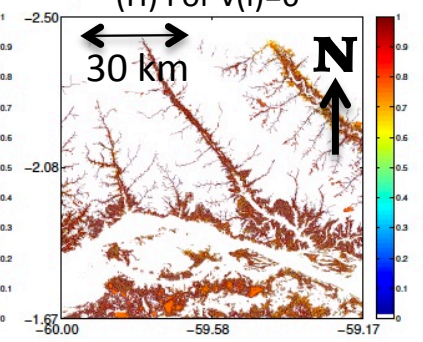

(l) For $v(i)=8$

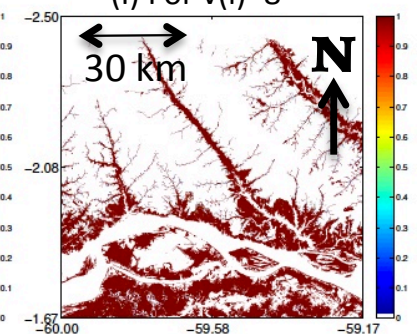

Figure 6: Maps of the $P[I(i)=1 \mid V(i)]$ (see text), before (left) and after (right) the interpolation in $n$, for $V(i)=0,2,4,6$, and 8 , from top to bottom. 
dataset is available in the two regions under study here (Camargue and Mekong), the full image global statistics will be used in the following of this paper.

\section{Filter 2}

For pixel $i$, with $V_{i}(O)$ inundated pixels in its neighbourhood:

$$
\begin{aligned}
& I(i)=1 \text { if } P\left[I(i)=1 \mid V_{i}(O)\right]>\left(1-\tau_{2}\right) \\
& I(i)=0 \text { if } P\left[I(i)=1 \mid V_{i}(O)\right]<\tau_{2}
\end{aligned}
$$

where $\tau_{2}$ is a threshold, to be optimised. This filter favours the inundation of pixels that are surrounded by inundated pixels, and favours the no-inundation of pixels surrounded by dry pixels.

Having a same neighbourhood constraint for the whole image is easier, but in some case, it is not optimal. For instance, a pixel with a change in landscape (e.g. rice paddy boundary) cannot be dealt optimally by a general constraint, but a neighbourhood constraint specific to this particular pixel will deal with local specificities by using a specific local conditional probability.

\subsection{Filter 3: Elevation}

This new filter identifies the inundated pixels in the neighbourhood, and then combines this information with topography-based constrains (Section 2.4). The basic idea is simple: If there is no inundation in the neighbourhood pixels $\mathcal{N}(i)$ :

- If pixel $i$ has lower chances to be inundated (higher elevation) than its surrounding, then it must not be inundated;

- If pixel $i$ has higher chances to be inundated (lower elevation), then nothing can be said so no change should be performed.

If there are some inundated pixels in neighbourhood $V(i)$, then this "reference" can be used to decide if pixel $i$ should be inundated or not:

- If pixel $i$ has lower chances to be inundated (higher elevation than its surrounding), then no change should be performed;

- If pixel $i$ has higher chances to be inundated (lower elevation than its surrounding), then it must be inundated. 


\section{Filter 3}

For pixel $i$, let $V(i)$ be the size of the inundated pixels $\overline{\mathcal{N}}(i)$ in the neighbourhood of $i$ :

- If $V(i)=0$ (i.e. no inundated pixel in $i$ neighbourhood):

$I(i)=0$ if $\operatorname{elev}(i)>\max (\operatorname{elev}(\mathcal{N}(i)))$

$I(i)$ not changed if $\operatorname{elev}(i) \leq \max (\operatorname{elev}(\mathcal{N}(i)))$

- Else if $V(i)>4$ :

$I(i)=1$ if $\operatorname{elev}(i) \leq \min (\operatorname{elev}(\overline{\mathcal{N}}(i)))$

$I(i)$ not changed if elev $(i)>\min (\operatorname{elev}(\overline{\mathcal{N}}(i)))$

In this filter, the "mean" could had been used instead of the "max" and the threshold $V(i)>4$ could be optimised too. The choices were made here based on a trial and error approach, and the application of this filter to another database would require optimising these parameters.

In Westerhoff et al. (2013), the HAND (Height Above the Nearest Drainage area) is used as a pre-processing to mask pixels with low probability of being inundated. The Filter 3 here is also based on topography, but it is a slightly different: (1) only local pixels are used, where the HAND variable can be related to distant pixels, and (2) in Westerhoff et al. (2013) the information is used as a pre-processing step and independently from the observation, when the topography a priori is used in combination with the observations (SAR or MODIS). This shows however how multiple forms of a priori information for the presence of surface waters can be derived from the same information, and how it is easy to combine them to optimise the detection of surface waters.

\subsection{Filter 4: Floodability Index}

This filter is based on the same idea but it uses the Floodability Index $(F I)$ of Section 2.5 instead of the elevation. This $F I$ is using topography information as for Filter 3 but a sophisticated work was done (neural network probabilistic model, exploitation of other topography information in addition to the elevation only) in order to obtain an index linked to the presence of surface water: 


\section{Filter 4}

For pixel $i$ :

- If $V(i)=0$, i.e. no inundated pixel in the neighbourhood $\mathcal{N}(i)$ :

$I(i)=0$ if $F I(i)<\min (F I(\mathcal{N}(i)))$

$I(i)$ not changed if $F I(i) \geq \min (F I(\mathcal{N}(i)))$

- Else if $V(i)>4$ :

$I(i)=1$ if $F I(i) \geq \min (F I(\overline{\mathcal{N}}(i)))$

$I(i)$ not changed if $F I(i)<\min (F I(\overline{\mathcal{N}}(i)))$

\section{De-noising results}

De-noising an image is more difficult than filling missing-data because it is not known a priori where the data have been corrupted or not; so when correcting for the noise, it is possible to modify a perfectly good observation. Furthermore, when using an a priori information for reducing the noise, the weight of the actual observation $O$ is reduced: a good compromise needs to be found between these two sources of information. The tests that will be conducted here will be based on specific data and some assumptions so the results obtained could change in other conditions. The goal of these experiments is to present various forms of a priori information that can be used in the de-noising of inundation maps.

\subsection{Experiments on "synthetic" data over Amazon}

The data used here are real observations (GIEMS-D3, Section 2.2) but the noise that is added for the de-noising experiments is synthetic. The original GIEMS-D3 image $I$ (i.e. target of the de-noising filters) is represented in the first map of Fig. 7(A). The corresponding noisy observed image $O$ is then presented in the second map (B) with a $30 \%$ noise level, i.e. $30 \%$ of the pixels have been switched to simulate noise.

Note first that a "symmetry" difficulty can appear in these experiments. In the original map I: $16.7 \%$ of the pixels are permanently inundated; $52.3 \%$ of the pixels are permanently dry; and $31 \%$ are transitory pixels. If $30 \%$ of noise is present in the image (i.e. $30 \%$ of $0 / 1$ switching values) then in the noisy image: $0.30 \times 0.167=5 \%$ are erroneously dry pixels $(\mathrm{FN})$, and $0.30 \times 0.523=15.69 \%$ are erroneously wet pixels $(\mathrm{FP})$. Therefore, a spatial 

satisfactory solution.

The de-noising results from the filters presented in Section 3 are presented in Fig. 7, and the numerical results are provided in Tab. 3 .

- Filter $1 a$ - The Filter 1a with threshold $\tau_{1 a}=0$ improves considerably the image (from and $1 \mathrm{~b})$. It is based here on the number of surrounding inundated pixels $V(i)$. The optimised threshold $\tau_{2}=0.3$ is a satisfactory value, it decreases the score from $10.4 \%$ to $8.8 \%$, see Filter III in Fig. 7(E).

- Filter 3 - Filter 3 uses topography information in the form of elevation. When used 
(A) ORIGINAL

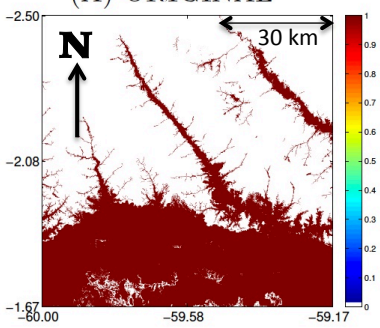

(D) Filter II

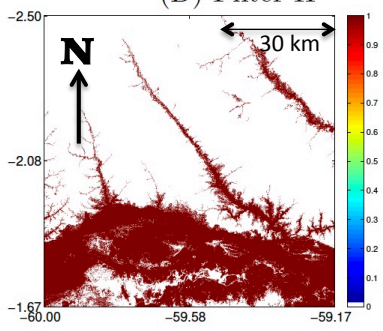

(B) NOISY $(30 \% \mathrm{SWITCH})$

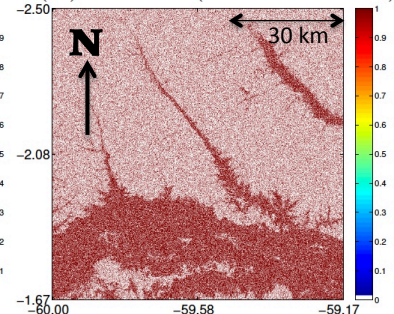

(E) Filter III

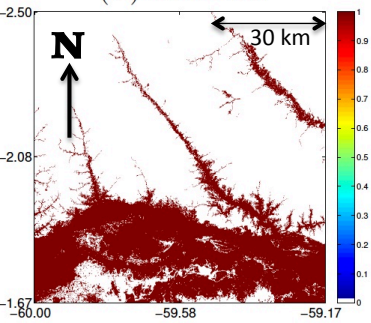

(G) Filter V

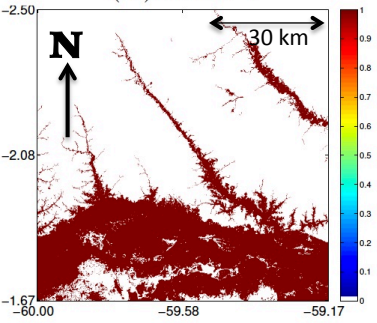

(C) Filter I

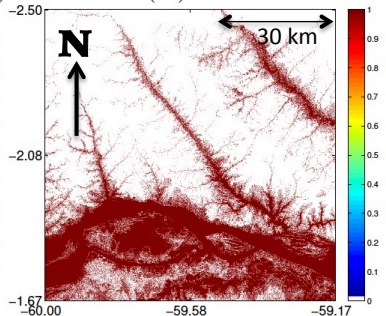

(F) Filter IV

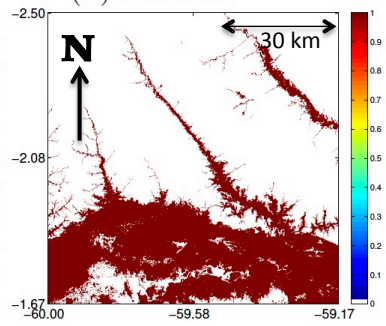

Figure 7: De-noising results, from left to right and top to bottom : Noise-free image (A), noisy image (B), and de-noising results from Filter I (C), Filter II (D), Filter III (E), Filter IV (F), and Filter V (G). See Tab. 3 for the definition of the labels in this figure.

\begin{tabular}{|c|c|c|c|c|c|}
\hline & $\begin{array}{l}\text { Fig. } 7 \\
\text { label }\end{array}$ & Thresholds & $\begin{array}{l}\mathrm{Nb} \text { residual } \\
\text { errors }\end{array}$ & $\begin{array}{l}\text { Percent. residual } \\
\text { error / total pixels }\end{array}$ & $\begin{array}{l}\text { Percent. res. error } \\
\text { / transitory pixels }\end{array}$ \\
\hline Noisy image & (B) & - & 300160 & $30.0 \%$ & - \\
\hline Filter 1a & & $\tau_{1 a}=0$ & 93193 & $9.3 \%$ & $31.0 \%$ \\
\hline Filter $\mathbf{I}=1 \mathrm{a}$ & (C) & $\tau_{1 a}=0.35$ & 30306 & $3.0 \%$ & $10.1 \%$ \\
\hline Filter $\mathbf{I I}=1 \mathrm{a}+1 \mathrm{~b}$ & (D) & $\tau_{1 a}=0 \mid \tau_{1 b}=0.1$ & 31113 & $3.1 \%$ & $10.4 \%$ \\
\hline \multicolumn{6}{|l|}{ Filter $\mathbf{I I I}=$} \\
\hline $1 a+1 b+2$ & $(\mathrm{E})$ & $\tau_{1 a}=0\left|\tau_{1 b}=0.1\right| \tau_{2}=0.3$ & 26534 & $2.6 \%$ & $8.8 \%$ \\
\hline Filter $1 \mathrm{a}+1 \mathrm{~b}+3$ & & $\tau_{1 a}=0\left|\tau_{1 b}=0.1\right| \tau_{3}=1$ & 25340 & $2.5 \%$ & $8.4 \%$ \\
\hline \multicolumn{6}{|l|}{ Filter $\mathbf{I V}=$} \\
\hline $1 \mathrm{a}+1 \mathrm{~b}+2+3$ & $(\mathbf{F})$ & $\tau_{1 a}=0\left|\tau_{1 b}=0.1\right| \tau_{2}=0.2 \mid \tau_{3}=1$ & 21858 & $2.2 \%$ & $7.3 \%$ \\
\hline Filter $1 \mathrm{a}+1 \mathrm{~b}+4$ & & $\tau_{1 a}=0\left|\tau_{1 b}=0.1\right| \tau_{4}=1$ & 26182 & $2.6 \%$ & $8.7 \%$ \\
\hline $\begin{array}{l}\text { Filter } 1 \mathrm{a}+1 \mathrm{~b}+2+4 \\
\text { Filter } \mathbf{V}=\end{array}$ & & $\tau_{1 a}=0\left|\tau_{1 b}=0.1\right| \tau_{2}=0.2 \mid \tau_{4}=1$ & 19797 & $2.0 \%$ & $6.6 \%$ \\
\hline $1 \mathrm{a}+1 \mathrm{~b}+2+3+4$ & (G) & $\tau_{1 a}=0\left|\tau_{1 b}=0.1\right| \tau_{2}=0.2\left|\tau_{3}=1\right| \tau_{4}=1$ & 18901 & $1.9 \%$ & $6.3 \%$ \\
\hline
\end{tabular}

Table 3: De-noising results of the five filters in terms of residual errors, percentage of residual errors in the full image, and percentage residual errors in the working space (i.e. not permanently wet or dry pixels). The Fig. 7 sub-labels of the five chosen filtered images is indicated in bold in the second column. 
a priori information for de-noising than Filter 3 (with score 8.8\%). When combined with Filter 3, the score decreases further and significantly (statistically, p-value=0.003) from 8.4 to $7.3 \%$, see Filter IV in Fig. 7(F). Significant has two meanings here. First, significant in statistics means that there are numerous data so if a difference is seen between two experiments, it is not random, there is a difference in information between the two filters. Second, the term significant is used here because a $1 \%$ increase in quality (from 8.4 to $7.3 \%$ ) is important because it is harder to improve a filter when it is already good. A $1.1 \%$ decrease represents almost $13.1 \%$ (from 8.4 to $7.3 \%$ ) of the errors in this case. Furthermore, in the $30 \mathrm{~m}$ resolution maps we are dealing here, this $1.1 \%$ decrease of errors translate into many pixels covering several $\mathrm{km}^{2}$ of ground area, become correctly classified.

- Filter 4 - Last a priori information is the Floodability Index used by Filter 4 . When combined with Filter 2, Filter 4 has a score of $6.6 \%$. When combined with Filter 3, the remaining error rate decreases even further at 6.3\%, see Filter V in Fig. $7(\mathrm{G})$. This shows that the FI is better information than the neighbourhood or elevation.

This set of experiments shows how it is possible to combine several a priori information by using together, hierarchically, several spatial filters. The erroneous pixels can be decreased in the whole image from $30 \%$ to $1.9 \%$ (in the "total" space), and the erroneous pixels in the transitory pixels from $31 \%$ to $6.3 \%$ (in the "work" space), see last line of Tab. 3 . The order in which these filters are stacked is important: some aberrant pixels can be corrected using some simple a priori information (e.g. permanently dry or wet pixels), then more sophisticated information can be used to improve further the solution.

\subsection{Experiments on $S A R$ data over Vietnam}

In these experiments, the Sentinel SAR Instantaneous image introduced in Section 2.1.1 is used. It was shown in that section how this image is corrupted by noise. The goal of the filters will be to reduce this noise and it will be possible to analyse visually the image to see the quality of the results. In order to have some quantitative measure of quality, the results will also be compared to the Reference image, even if it is dangerous to directly compare these images due to the acquisition-time differences.

- Filter $1 a$ - Tab. 4 provides the confusion matrix of the Filter 1 for $\tau_{1 a}$ from 0 to 0.35 . The highest this threshold, the highest our confidence on the GSWO a priori probability is. The number of erroneous pixels goes from 715,556 for the Instantaneous image (Tab. 1) to a 
range of $[692,012 / 369,072]$, depending on $\tau_{1 a}$. This represents a decrease of the erroneous pixels close to $50 \%$ (part of the remaining differences is related to the time-difference). It

\begin{tabular}{|c|c|c|}
\hline \multicolumn{3}{|c|}{ Filter 1a } \\
\hline$\tau_{1 a}=\mathbf{0 . 0 0}$ & $\mathrm{N}=25,516,150$ & $\mathrm{P}=1,473,090$ \\
\hline \multirow[t]{2}{*}{$\mathrm{N}=25,397,582$} & $\mathrm{TN}=25,110,860$ & $\mathrm{FP}=286,722$ \\
\hline & $98.41 \%$ & $19.46 \%$ \\
\hline \multirow[t]{2}{*}{$\mathrm{P}=1,591,658$} & $\mathrm{FN}=405,290$ & $\mathrm{TP}=1,186,368$ \\
\hline & $1.59 \%$ & $80.54 \%$ \\
\hline Errors $=692,012$ & $2.56 \%$ / work & $2.29 \% /$ total \\
\hline \multirow[t]{5}{*}{$\tau_{1 a}=\mathbf{0 . 1 5}$} & $\mathrm{N}=25,375,103$ & $\mathrm{P}=1,614,137$ \\
\hline & $\mathrm{TN}=25,170,379$ & $\mathrm{FP}=227,203$ \\
\hline & $99.19 \%$ & $14.08 \%$ \\
\hline & $\mathrm{FN}=204,724$ & $\mathrm{TP}=1,386,934$ \\
\hline & $0.81 \%$ & $85.92 \%$ \\
\hline Errors $=431,927$ & $1.60 \% /$ work & $1.43 \%$ / total \\
\hline \multirow[t]{5}{*}{$\tau_{1 a}=\mathbf{0 . 3 0}$} & $\mathrm{N}=25,454,111$ & $\mathrm{P}=1,535,129$ \\
\hline & $\mathrm{TN}=25,241,263$ & $\mathrm{FP}=156,319$ \\
\hline & $99.16 \%$ & $10.18 \%$ \\
\hline & $\mathrm{FN}=212,848$ & $\mathrm{TP}=1,378,810$ \\
\hline & $0.84 \%$ & $89.82 \%$ \\
\hline Errors $=369,167$ & $1.36 \%$ / work & $1.22 \% /$ total \\
\hline
\end{tabular}

Table 4: De-noising statistics for the Filter 1a (based on pixel probability from GSWO) for threshold $\tau_{1 a}=$ 0, 0.15, and 0.3. True Negative (TN), False Positive (FP), False Negative (FN), and True Positive (TP) are provided, together with the number of erroneous pixels. "Work" is for working space (pixels not permanently inundated or dry).

can be seen that by increasing $\tau_{1 a}$, the number of False FN decreases, and then starts to increase; but that the FP are monotonically decreasing. Fig. 8 shows the evolution of the total, FN, and FP numbers with $\tau_{1 a}$. The threshold $\tau_{1 a}=0.15$ is chosen in the following as a good compromise. The false positive errors still continue after this threshold, but a too large $\tau_{1 a}$ means a very high confidence on GSWO. This would be justified in some regions (large uniform regions as inside rivers), but this would not be true in all areas such as floodplains 
or vegetated areas not well described by GSWO. Using only the diagnostics such as FP, FN, $\mathrm{TP}$ and TN percentages is very valuable of course, but understanding of the information content of all the datasets is also important. The GSWO a priori information appears to be of good quality in this part of the Mekong region and for this time of year because the surface waters are mostly rivers, and GSWO, by using visible data, is able to detect such open waters. Over other regions, the quality of this a priori could be of lower quality.

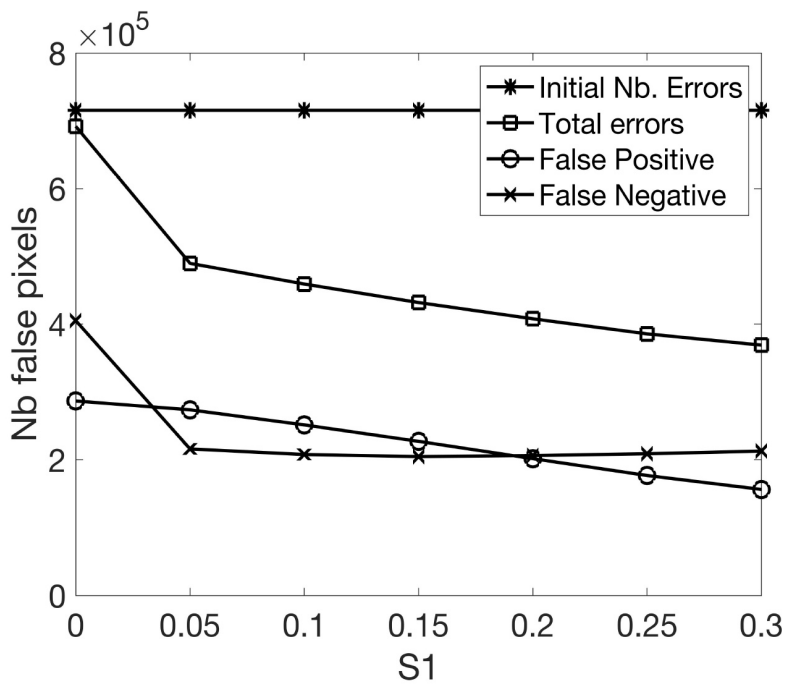

Figure 8: Evolution of the Total, False Positives, and False Negative errors with Filter 1a with increasing $\tau_{1 a}$, for the Mekong image de-noising.

Fig. 9 represents the GSWO a priori information, together with the Instantaneous image and the de-noising using $\tau_{1 a}=0.15$. The use of the a priori information provides uniform pixel values inside the rivers, because the GSWO has uniform probabilities in these regions.

- Filter 2 - By using simple and uniform statistics on the 8 neighbours of a pixel $(\operatorname{Prob}(I)(i)=$ $1 \mid(V(i)=n))$, it allows decreasing the number of erroneous pixels from 715,556 in the Instantaneous image to 597,034 (about 17\% decrease), after 4 iterations (with $\tau_{2}=0.2$ ). Results are provided in Tab. 5. Even after this 17\% decrease), the FP score is still high at $18.91 \%$. This can be explained by the fact that the neighbourhood statistics are done globally in the ensemble of all pixels because no Sentinel historical time series was available to build a statistics for each pixel. A possible extension of this work could be to divide the pixels in several specific groups (for instance pixels close to permanently inundated, close 
(A) GSWO

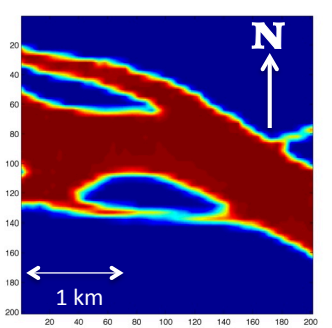

(B) Instantaneous

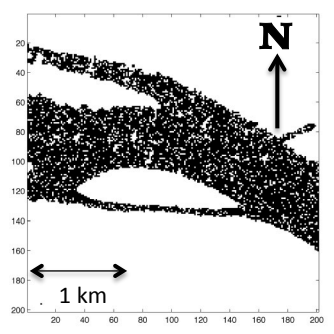

(C) $s_{1 a}=0.15$

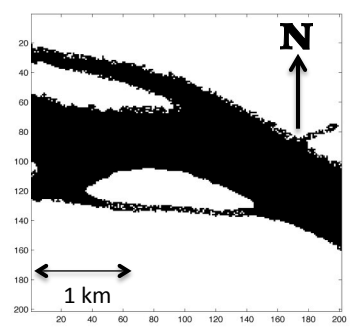

(D) Reference

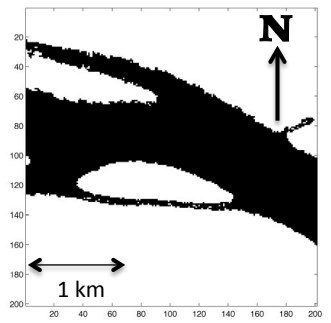

Figure 9: De-noising results for Filter 1a (i.e. pixel probability from GSWO) over one zoom of the Mekong region (see black square in Fig. 1). From left to right: GSWO water occurence (A), Instantaneous noisy image (B), filtering with $\tau_{1 a}=0.15(\mathrm{C})$, and Reference (D) image. 
to never inundated, or transitory pixels) and to perform a statistics for each one of these groups. Similarly to Fig. 9, the de-noised map from Filter 2 (not shown) has a decreased number of FN pixels inside the river, which is the aim of such filter. FP pixels are difficult to correct and numerous errors remain (18.91\%). In fact, it is difficult to suppress the FP pixels at the edge of the rivers because the neighbourhood constrain focuses here on pixels with low number of inundated pixels. Furthermore, it cannot be known if the river edges have changed during the 40-day antecedent period of the Reference image.

- Filter 3 - Tab. 5 shows the de-noising statistics for the DEM-based Filter 3 (with $\tau_{3}=1$ ). The number of errors decrease from 715,556 to 624,492 (a $12.7 \%$ decrease). Results are mitigated for this filter. It should be noted however that DEM information are useful only over land, not over water. Noise errors in this image are focused over the open waters, so these results are not surprising.

- Filter 4 - Tab. 5 shows the de-noising statistics for the FI-based Filter 4 (with $\tau_{4}=1$ ). Number of errors decrease from 715,556 to 550,568 (a 23\% decrease of the errors). Results are better than those from the DEM (Filter 3), although the FI in entirely defined by the DEM. This means that the FI is able to obtain a more direct information on flooding than the elevation alone. Again, the FI provides information over land, and not over the rivers. So this type of information should be better for images with errors over land.

- Combining filters - The combination of the previously introduced Filters is tested here. Tab. 6 shows the de-noising statistics. Number of errors decrease from 715,556 to 422,913 (a significant decrease by $40.9 \%$ of the errors). Note that errors are measured based on the Reference image but that differences are inevitable between the Reference and the Instantaneous images. So part of the remaining errors are explained not by a default spatial interpolation, but by these Instantaneous/Reference differences.

More than these statistical diagnostics, a reassuring fact is that all the filters are acting in agreement with what they were designed to do. This means that the constraints that were specified (historical, neighbourhood-based, etc.) are sound and that the way they are exploited in the filter is working properly. If other constrains are requested by a particular application, it would be possible to design other filters. 


\begin{tabular}{|r|rr|}
\hline \multicolumn{3}{|r|}{ Filter 2: Neighbour, $\tau_{2}=0.3$} \\
\hline Iteration 1 & $\mathrm{N}=25,416,672$ & $\mathrm{P}=1,572,568$ \\
\hline $\mathrm{N}=25,397,582$ & $\mathrm{TN}=25,096,872$ & $\mathrm{FP}=300,710$ \\
& $\mathrm{TN}=98.74 \%$ & $19.12 \%$ \\
$\mathrm{P}=1,591,658$ & $\mathrm{FN}=319,800$ & $\mathrm{TP}=1,271,858$ \\
& $1.26 \%$ & $80.88 \%$ \\
\hline Errors=620,510 & $2.30 \% /$ work & $2.06 \% /$ total \\
\hline Iteration 4 & $\mathrm{N}=25,389,590$ & $\mathrm{P}=1599650$ \\
\hline Ref Neg= & $\mathrm{TN}=25,095,069$ & $\mathrm{FP}=302,513$ \\
& $98.84 \%$ & $18.91 \%$ \\
Ref Pos $=$ & $\mathrm{FN}=294,521$ & $\mathrm{TP}=1,297,137$ \\
& $1.16 \%$ & $81.09 \%$ \\
\hline Errors=597,034 & $2.21 \% /$ work & $1.98 \% /$ total \\
\hline
\end{tabular}

\begin{tabular}{|c|c|c|}
\hline \multicolumn{3}{|c|}{ Filter 3: DEM, $\tau_{3}=1$} \\
\hline Filter 3 & $\mathrm{~N}=25,398,882$ & $\mathrm{P}=1,590,358$ \\
\hline \multirow[t]{2}{*}{$\mathrm{N}=25,397,582$} & $\mathrm{TN}=25,397,582$ & $\mathrm{FP}=311,596$ \\
\hline & $\mathrm{TN}=98.77 \%$ & $19.59 \%$ \\
\hline \multirow[t]{2}{*}{$\mathrm{P}=1,591,658$} & $\mathrm{FN}=312,896$ & $\mathrm{TP}=1,278,762$ \\
\hline & $1.23 \%$ & $80.41 \%$ \\
\hline Errors $=624,492$ & $2.31 \%$ / work & $2.07 \%$ / total \\
\hline \multicolumn{3}{|c|}{ Filter 4: Flood. Ind., $\tau_{4}=1$} \\
\hline Filter 4 & $\mathrm{~N}=25,233,574$ & $\mathrm{P}=1,755,666$ \\
\hline \multirow[t]{2}{*}{$\mathrm{N}=25,397,582$} & $\mathrm{TN}=25,040,294$ & $\mathrm{FP}=357,288$ \\
\hline & $\mathrm{TN}=99.23 \%$ & $20.35 \%$ \\
\hline \multirow[t]{2}{*}{$\mathrm{P}=1,591,658$} & $\mathrm{FN}=193,280$ & $\mathrm{TP}=1,398,378$ \\
\hline & $0.77 \%$ & $79.65 \%$ \\
\hline Errors $=550,568$ & $2.04 \%$ / work & $1.83 \% /$ total \\
\hline
\end{tabular}

Table 5: De-noising statistics for the Filter 2 (Neighbourhood-based) after iteration 1, and 4; for the Filter 3 (DEM based); and for the Filter 4 (Floodability Index based). 


\begin{tabular}{|c|c|c|}
\hline \multicolumn{3}{|c|}{ Combination } \\
\hline Filter $1 \mathrm{a} / \tau_{1}=0.15$ & $\mathrm{~N}=25,375,103$ & $\mathrm{P}=1,614,137$ \\
\hline & $\mathrm{TN}=25,170,379$ & $\mathrm{FP}=227,203$ \\
\hline & $99.19 \%$ & $14.08 \%$ \\
\hline & $\mathrm{FN}=204,724$ & $\mathrm{TP}=1,386,934$ \\
\hline & $0.81 \%$ & $85.92 \%$ \\
\hline Errors $=431,927$ & $1.60 \%$ / work & $1.43 \% /$ total \\
\hline+ Filter 2 & $\mathrm{~N}=25,357,312$ & $\mathrm{P}=1,631,928$ \\
\hline & $\mathrm{TN}=25,166,616$ & $\mathrm{FP}=230,966$ \\
\hline & $99.25 \%$ & $14.15 \%$ \\
\hline & $\mathrm{FN}=190,696$ & $\mathrm{TP}=1,400,962$ \\
\hline & $0.75 \%$ & $85.85 \%$ \\
\hline Errors $=421,662$ & $1.56 \%$ / work & $1.40 \% /$ total \\
\hline+ Filter 3 & $\mathrm{~N}=25,352,672$ & $\mathrm{P}=1,636,568$ \\
\hline & $\mathrm{TN}=25,164,951$ & $\mathrm{FP}=232,631$ \\
\hline & $99.26 \%$ & $14.22 \%$ \\
\hline & $\mathrm{FN}=187,721$ & $\mathrm{TP}=1,403,937$ \\
\hline & $0.74 \%$ & $85.78 \%$ \\
\hline Errors $=420,352$ & $1.56 \%$ / work & $1.40 \% /$ total \\
\hline+ Filter 4 & $\mathrm{~N}=25,298,093$ & $\mathrm{P}=1,691,147$ \\
\hline & $\mathrm{TN}=25,136,381$ & $\mathrm{FP}=261,201$ \\
\hline & $99.36 \%$ & $15.44 \%$ \\
\hline & $\mathrm{FN}=161,712$ & $\mathrm{TP}=1,429,946$ \\
\hline & $0.64 \%$ & $84.55 \%$ \\
\hline Errors $=422,913$ & $1.57 \%$ / work & $1.40 \% /$ total \\
\hline
\end{tabular}

Table 6: De-noising statistics for the combination of Filters. 


\section{Missing-data results}

Filling missing pixels using spatial interpolation techniques over inundation maps is easier than the de-noising problem of the previous section because the location of missing-data is known, and there is no risk to wrongly modify correctly observed pixels. Furthermore, the filling of missing-data is faster in terms of computations because the filters need to be applied only on the missing pixels, not on the full image.

\subsection{Missing pixel results on "synthetic" data over Amazon}

Fig. 10(A) shows the original GIEMS-D3 image $I$, and the corresponding image with black dots in the location of $15 \%$ of missing pixels (B). The solutions provided by Filters 1a, 1b, 2, 3, and 4 are then represented. Filter $1 \mathrm{a}$ is with a threshold value $\tau_{1 a}=0$.
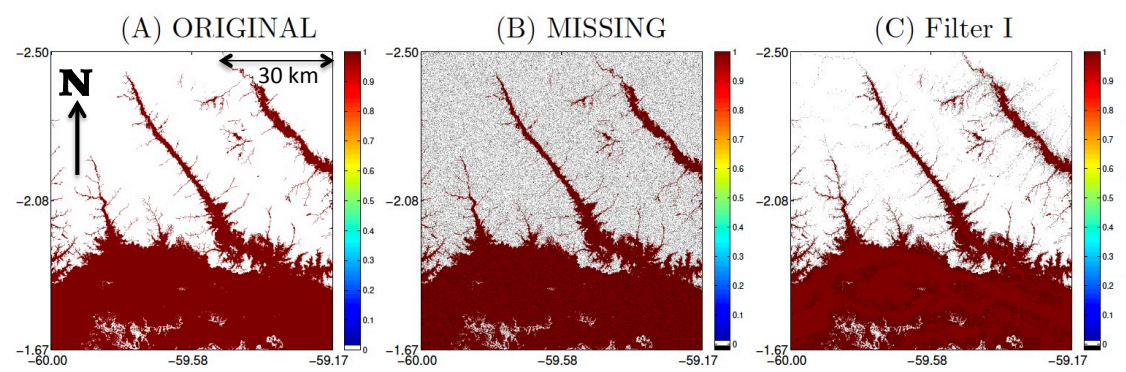

(D) Filter II

(E) Filter III

(F) Filter IV
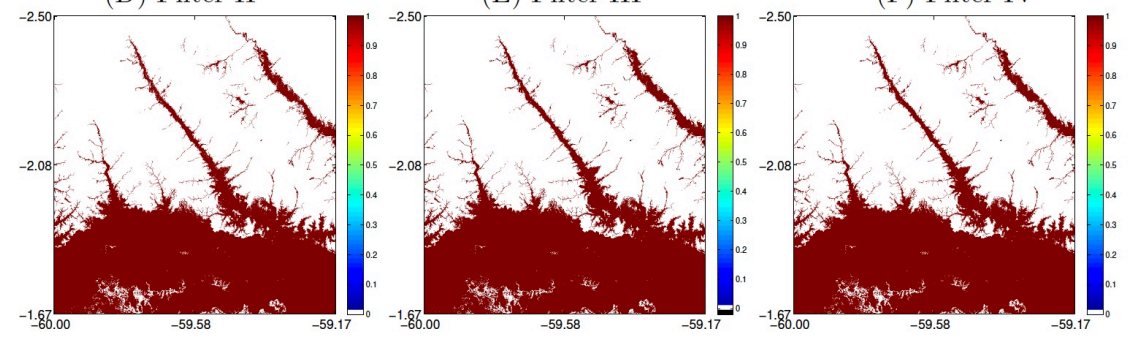

(G) Filter V

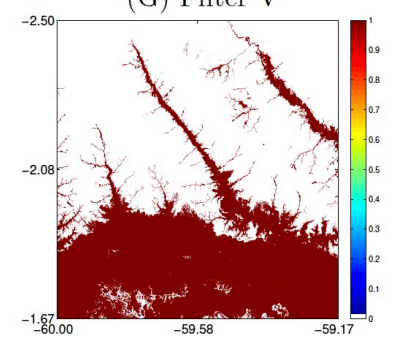

Figure 10: Filling results over an Amazon region. From left to right and top to bottom : Original image $I$ (A), image $O$ with missing pixels (B), and filling results from Filter I (C), Filter II (D), Filter III (E), Filter IV (F), and a Filter V (G) combination. 
When using these filters, all the missing pixels are not filled. By increasing the threshold of each filters, more pixels are processed, but the errors in the filled pixels increase. Each application would require a different balance based on the tolerance to errors. The numerical results are synthesised in Tab. 7. It can be seen that it is possible to fill in a considerable number of the missing pixels: from $31 \%$ for Filter 1 a with $\tau_{1 a}=0$, to $0.6 \%$ when combining the five filters in Filter V, see Filter I in Fig. 10(C). The erroneous filling stays at a low value: from $0 \%$ with Filter 1a to $0.4 \%$ when combining the five filters (Fig. 10(G)). In order to fill in all the pixels, the last line of Tab. 7 uses the combined Filter $\mathrm{V}$ with $\tau_{1 a}=0$, $\tau_{1 b}=0, \tau_{2}=0.2, \tau_{3}=1$, and $\tau_{4}=1$, but with a post-processing by Filter $1 \mathrm{~b}$ with threshold $\tau_{1 b}=0.5$ to fill in the 961 remaining pixels: the erroneous pixels increase from 540 to 960 , it means that 420 errors were present over the remaining 961 ambiguous pixels. The filters have therefore a satisfactory behaviour with coherent results.

\begin{tabular}{|c|c|c|c|c|c|c|}
\hline & $\begin{array}{l}\text { Fig. } 7 \\
\text { sub-label }\end{array}$ & $\begin{array}{l}\text { Thresholds } \\
\left(\tau_{1 a}, \tau_{1 b}, \tau_{2}, \tau_{3}, \tau_{4}\right)\end{array}$ & $\begin{array}{r}\mathrm{Nb} \\
\text { missing } \\
\end{array}$ & $\begin{array}{r}\text { Percent. } \\
\text { missing left }\end{array}$ & $\begin{array}{r}\mathrm{Nb} \\
\text { errors }\end{array}$ & $\begin{array}{r}\text { Percent. } \\
\text { errors }\end{array}$ \\
\hline Image & (B) & - & 150000 & $100.0 \%$ & - & - \\
\hline Filter 1a & & $\tau_{1 a}=0$ & 46728 & $31.2 \%$ & 0 & $0.0 \%$ \\
\hline Filter I & $(\mathrm{C})$ & $\tau_{1 a}=0.1$ & 22417 & $15.0 \%$ & 459 & $0.3 \%$ \\
\hline Filter II & (D) & $\tau_{1 a}=0 / \tau_{1 b}=0.1$ & 7887 & $5.3 \%$ & 159 & $0.1 \%$ \\
\hline Filter III & $(\mathrm{E})$ & $\tau_{1 a}=0 / \tau_{1 b}=0 / \tau_{2}=0.1$ & 1994 & $1.3 \%$ & 210 & $0.1 \%$ \\
\hline Filter 3 & & $\tau_{1 a}=0 / \tau_{3}=1$ & 19592 & $13.0 \%$ & 503 & $0.3 \%$ \\
\hline Filter IV & $(\mathbf{F})$ & $\tau_{1 a}=0 / \tau_{1 b}=0 / \tau_{2}=0.2 / \tau_{3}=1$ & 990 & $0.7 \%$ & 535 & $0.3 \%$ \\
\hline \multirow[t]{3}{*}{ Filter 4} & & $\tau_{1 a}=0 / \tau_{4}=1$ & 20595 & $13.7 \%$ & 3 & $0.0 \%$ \\
\hline & & $\tau_{1 a}=0 / \tau_{1 b}=0 / \tau_{2}=0.2 / \tau_{4}=1$ & 1335 & $0.9 \%$ & 338 & $0.2 \%$ \\
\hline & & $\tau_{1 a}=0 / \tau_{1 b}=0 / \tau_{2}=0.2 / \tau_{3}=1 / \tau_{4}=1$ & 961 & $0.6 \%$ & 540 & $0.4 \%$ \\
\hline Filter V & $(\mathrm{G})$ & $\tau_{1 a}=0 \tau_{1 b}=0 \tau_{2}=0.2 \tau_{3}=1 \tau_{4}=1+\tau_{1 b}=0.5$ & 0 & $0 \%$ & 960 & $0.6 \%$ \\
\hline
\end{tabular}

Table 7: Filling results of the combined filters tested over the Amazon region: number and percentage of missing pixels, and number and percentage of errors in "the working space" (i.e. transitory pixels). The Fig. 7 sub-labels of the five selected filtered images is indicated in bold in the second column.

\subsection{Large-area missing-data on visible Sentinel 2 data over Camargue}

The a priori information becomes essential for the filling of missing-data because no direct observation is available in this case for the considered pixels except in the edge of the missing area (that can be far from it when the missing area is large). It is important to use an a priori information that is coherent with the observations otherwise, big discontinuities can appear between the observed and the interpolated areas. The ideal scenario is to use $a$ priori originating from the same instrument of the observations. This can be done when a historical record is available for that particular instrument. Unfortunately, this is not always 
the case, in particular here for the Sentinel 2 visible data over the Camargue region. An auxiliary a priori information needs to be used instead.

The most compatible source of information among the a priori databases presented in Section 2 is the GSWO dataset: It is directly related to the presence of surface waters (not like topography), it has similar spatial resolution, and for the Camargue region, the visible data from GSWO are coherent with the Sentinel 2 visible data. The GSWO will therefore be used in order to fill in the missing-data in the Sentinel 2 visible instantaneous data.

- Simple thresholding - The first spatial interpolation method to fill the missing-data (related to the cloud presence) uses a threshold on the GSWO occurence: $I=1$ if $G S W O>h$ and $I=0$ if $G S W O<h($ with $h=0.5)$. Fig. 11(B) represents the spatial interpolation performed over the GSWO a priori. No discontinuities can be seen between the non-cloudy and the cloudy regions, which means that the GSWO is an a priori information compatible with the visible Sentinel 2 data over this region. In the Interpolated-Reference image (E), the blue pixels indicate that the interpolated image is "drier" than the Reference (i.e. Interp-Ref=-1), and the red ones indicate that the interpolation is "wetter" (i.e. Interp-Ref=1).

A zoom is performed over a smaller area, in the same Camargue region (Fig. 12). It allows to better compare with the GSWO information also represented in this figure (A). Again, no discontinuity can be observed (E), stressing the quality of the a priori information over this region and for these data.

- Adaptative thresholding - In the previous approach, the water presence in a missing pixel was decided on a simple threshold $(h=0.5)$ over the $[0,1]$ occurrence range of the GSWO dataset. This threshold can be optimised for two reasons: (1) The occurence of water seen by the SAR Sentinel 1 or by Landsat on GSWO might differ. Their sensitivity is not the same. These differences might require and adjustment between the two sources of information. (2) The local "context" of the visible Sentinel 2 image, on non-missing pixels, can help adjusting this threshold. This is the principle of spatial interpolation: exploit the observed data where they are available to fill pixels where data are missing. If a region observed by the Sentinel 2 is wetter than usual, then more inundation should be allowed in its neighbourhood when doing the spatial interpolation in the missing area. Conversely, if a Sentinel 2 estimation region is drier, it should have a drying effect on the interpolation.

The principle of the adaptative thresholding presented here is based on the search of a 
(A) Instantaneous w. clouds

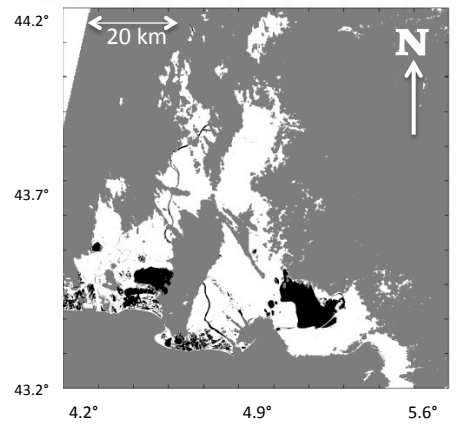

(D) Reference

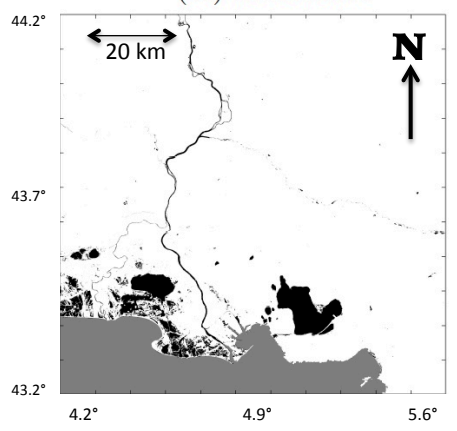

(B) Interp./Const

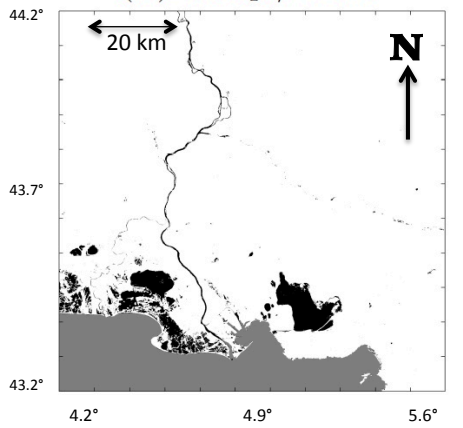

(E) Interp./Const. minus Ref.

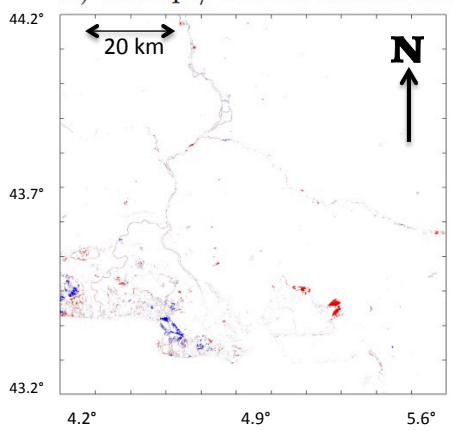

(C) Interp./Adapt.

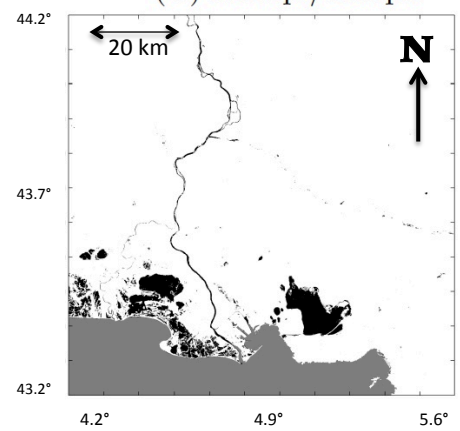

(F) Interp./Adapt. minus Ref.

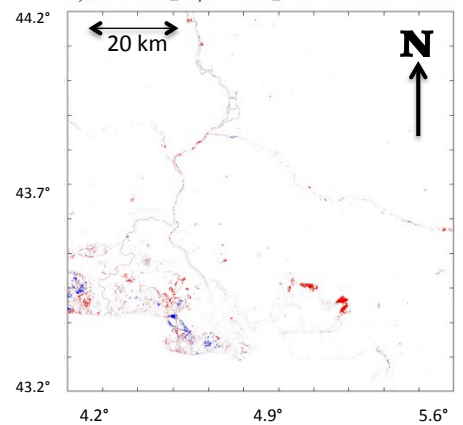

Figure 11: Data-missing filling results over Camargue. First row: Instantaneous (A), Interpolated with constant (B), and adaptative thresholds (C). Second row: Reference (D), Interpolated minus Reference with constant (E) and adaptative (F) thresholds. 
(A) GSWO

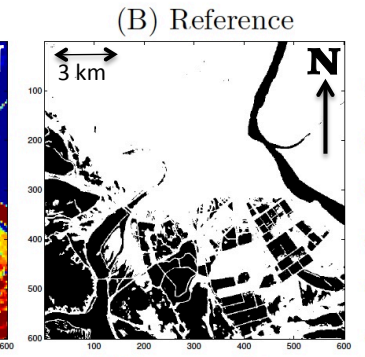

(C) Instantaneous-Ref

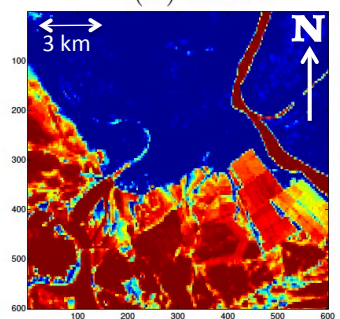

(E) Interp /Const

(D) Instantaneous
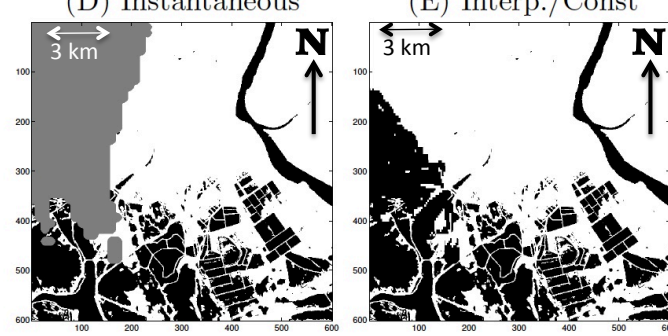

F) Interp./Const minus Ref
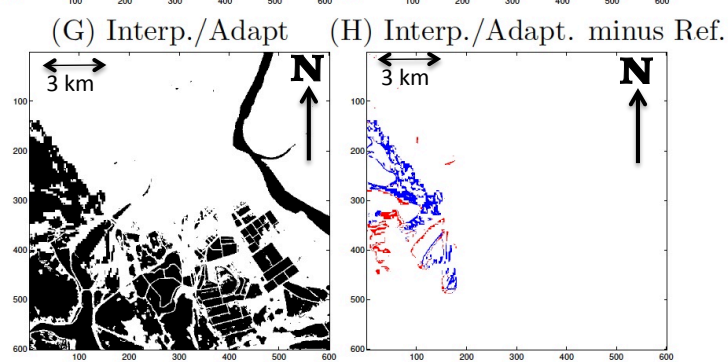

Figure 12: Zoom over the Camargue region for missing-data filling. First row: GSWO (A), Reference (B), and Instant./Const.-Reference (C). Second row: Instantaneous (D), Interp./Constant (E), and Interp./Const. minus Reference (F). Third row: Interp./Adaptative (H), and Interp./Adapt minus Reference (G). 
threshold $h$ that provides the best agreement between the visible Sentinel 2 observation and the GSWO occurence, for all the pixels over land with no clouds. For each non-missing pixel $i$ over land, the process is decomposed in the following steps:

- First, all the land and non-missing pixels in the neighbourhood of pixel $i$ are gathered into a vector: $V(i)$.

- The number of pixels $N(i)$ in $V(i)$ that are inundated is then obtained.

- The GSWO occurrences for the $V(i)$ pixels are gathered into a vector: $P=[G S W O(j), j \in$ $V(i)$ ] (see two upper graphs in Fig. 13(A and B)).

- $P$ is sorted in decreasing order: $P^{\prime}$ (see two lower graphs in Fig. 13(A and B)).

- The optimal threshold is given by $h=P^{\prime}(N(i))$ (same figure).

This adaptative threshold $h$ ensures that the use of the rule $G S W O(j) \geq h$ on $V(i)$ will provide the same number of inundated pixels $N(i)$ than in the observation map to be interpolated. Fig. 13(A and B) represents this process on two pixels (left and right), and the two obtained thresholds are 0.38 and 0.69 . This choice of the optimal threshold can be made only when inundated pixels are present in the neighbourhood (i.e. $N(i)>0$ ). In the other pixels (i.e. $N(i)=0$ ), the standard choice $h=0.5$ is adopted a priori.

For computational efficiency purpose, this adaptative threshold is required only at the edges of the cloudy missing areas, it is then possible to perform a spatial interpolation inside the missing areas. A bilinear interpolation technique can be used for instance. A so-called "natural-neighbour" interpolation is used.

Fig. 13(C) represents the map of the adaptative thresholds in all the non-cloudy pixels of the image, not only over the cloud-edges, for a better understanding. Optimised threshold can be $>0.5$ (meaning that locally, the observation is "dryer" than the GSWO a priori) or $<0.5$ (observation "wetter" than the GSWO a priori).

In agreement with Fig. 13 , the interpolation results of the adaptative method are presented in Fig. 12(G). The difference is that in a part of the image, the new interpolation gives less inundated pixels than the constant threshold approach because the Instantaneous visible Sentinel 2 image was dryer than the GSWO dataset on that day and location. When compared to the Reference image, some differences can be observed in Fig. 11(F). These 
(A) $\mathbf{h}=\mathbf{0 . 3 8}$
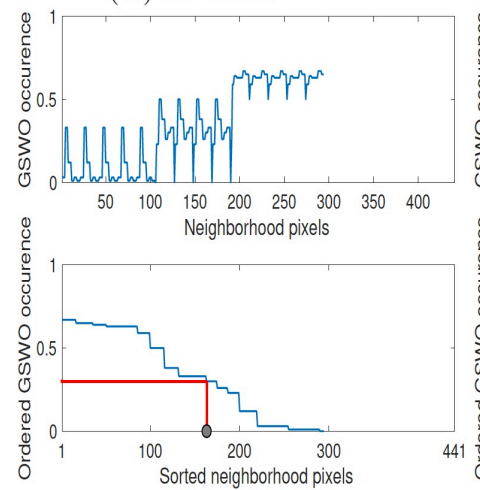

(B) $\mathbf{h}=\mathbf{0 . 6 9}$

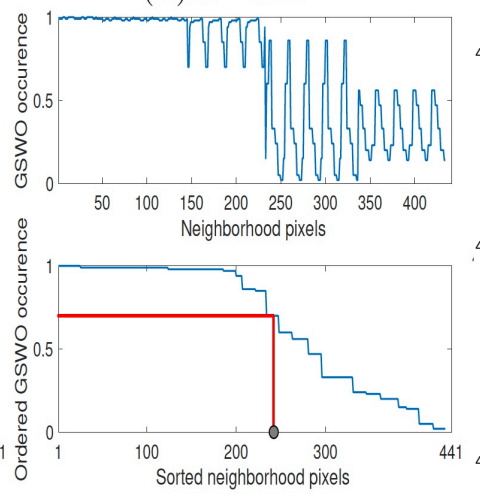

(C) Interpolated threshold $h$

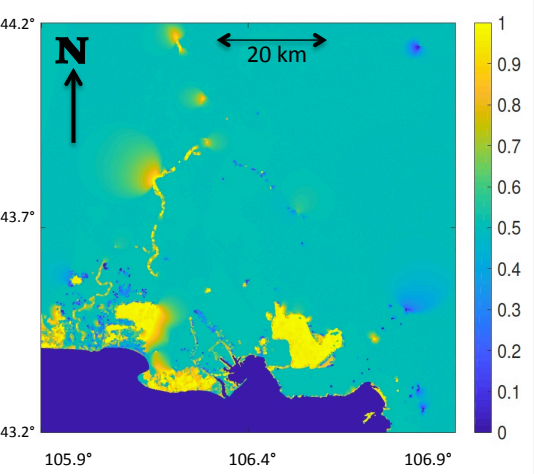

Figure 13: (A) and (B) columns: Choice of the adaptative threshold $h<G S W O(i)$ at the pixel level, over two pixels (A and B). Upper part: GSWO occurences on neighbourhood $V(i)$. Bottom part: Ordered occurrences and threshold definition. (C): Optimised adaptative threshold $h$. 
differences can be better seen in the zoom of Fig. 12(H). Although computationally slower, the Interp./Adapt. method allows to take into account in the spatial interpolation the specificities of the observations on that given date. This seems a better approach than using a simple constant threshold on the GSWO occurence.

\section{Discussion}

In this article, two regions over Camargue/France and Mekong/Vietnam have been considered. These two regions have been monitored respectively by visible and SAR observations from the Sentinel satellites. It was noticed that Mekong had missing-data due to the presence of clouds, and Camargue had noise due to instrument or retrieval errors.

Shen et al. (2019) state that automation and robustness have not been achieved yet for the detection of surface waters below the vegetation when using L-Band SAR observations (due to water-like surfaces, speckle noise, or geometric corrections). VIS/IR observations are contaminated too by clouds and vegetation presence. This means that misclassifications and missing data will be present in retrieved surface water maps. Human intervention to reduce over- or under-detection is often required (Shen et al., 2019).

Sophisticated retrieval approaches have been designed for some specific observations. For instance, change detection techniques can be used on time series of SAR backscatter coefficients (Giustarini et al., 2013, Matgen et al.). But the development of de-noising and missing-data filling techniques, as post-precessing step over the retrieved surface water maps, is more general and can be employed in any type of water maps. Pulvirenti et al. (2011a) use an object-oriented technique based on a dilution and an erosion steps to remove isolated groups of water pixels and small holes in water bodies. Another approach is to use a priori information (such as land cover, elevation, etc.) and exploit it within spatial filters (Pulvirenti et al., 2011b). This is the way tested here in this paper.

Four sources of a priori information on surface water have been exploited to reduce noise in surface water maps or fill missing values by spatial interpolation:

- Historical information at the pixel level: Advantage of this type of information is its simplicity. In this work, our source of information is the Landsat-based GSWO dataset (Pekel et al., 2016b). This type of information could be used as simple a priori for 
SWOT retrievals (even if some limitations of such datasets need to be considered Aires et al. 2018)

- Neighbouring information constrains based on historical information: This is a typical image processing approach. It generally requires a long historical record. Several forms of neighbouring constrains can be used based on the quality of the historical data.

- Constraints based on topography information: This a priori information is not based on surface water maps. However, it is evident hydrologically that surface waters are strongly constrained by topography.

- Constraints based on hydrology information: It has been shown that a floodability index indicating which pixels are more likely to be inundated in a region can be constructed based on topography information. For instance, in Fluet-Chouinard et al. (2015) and Aires et al. (2017), a floodability index was used to downscale inundation maps to a resolution of $90 \mathrm{~m}$.

It was investigated here if these four sources of a priori information can help post-processing corrupted surface water maps by reducing noise in retrieval and fill missing-data.

In the filling-data problem, the advantage is that the "working space" (i.e. the ensemble of pixels that are missing and that need to be complemented) is known. Most of the time in this context, the information content of the available observations is limited because available pixels can be far from the pixels to be filled in. So the a priori information is essential in this case. In the de-noising problem, the actual observations are more important because they are available for the considered pixels. The difficulty is related to fact that it is not known a priori which pixels are contaminated or not by noise, and by how much. A good compromise needs to be found between the observations and the a priori information.

De-noising - Four spatial filters have been introduced for the de-noising. It was shown how these filters can be combined to increase the quality of the surface water maps. Results for the Mekong region show that the filter from GSWO inundation probability is the most performant filter. However, the region that is considered includes mostly rivers (not floodplains), and it is known that GSWO is good to describe open waters. In other regions, with more vegetation, GSWO would be less reliable, and the use of the other filters might then become necessary. It was shown that the exploitation of our Floodability Index (based 
on topography) is more performant than the raw elevation from a DEM only. This was to be expected because this FI was designed to predict surface water presence in a better way than the DEM. The simple neighbourhood system exploited in Filter 2 was also not as performant than the FI. This is probably due to the lack of historical record that would allow obtaining statistical constraints for each pixel, and not uniformly for the whole image. This could be improved if a historical record is used instead of one image only (using another instrument than the Sentinel ones, or waiting after a mission launch for a time record long enough to build pixel-scale constraints). De-noising errors still remain but when looking in detail, the introduced filters follow well the job they were designed to accomplish (i.e. fill the holes inside the rivers, uniformizing spatially the water surfaces, limiting isolated inundated pixels, etc.).

Any instrument would have specific noise characteristics (from instrument, assumptions made, or retrieval errors). The de-noising experiments were conducted on SAR data, so a noise model is used for the synthetic experiments that are close to what is expected for that instrument. For another instrument, another model would be required.

Missing-data filling - Due to the available test data, two thresholding techniques were used on the a priori GSWO surface water occurrence to fill the missing areas due to the presence of clouds. Again, the GSWO seems to be a good source of a priori information, for the same reasons as for the de-noising. Two versions of this approach were considered: one with a constant threshold, and another with an adaptative one. The adaptative thresholding allows to better consider the Sentinel 2 visible/GSWO differences, and to use the specific conditions of the SAR observation to be interpolated (with drier or wetter local areas).

In the large-missing-area case, observations are not as important as in de-noising because the observations (non-cloudy pixels) can be very far from the missing pixels. Therefore, the a priori information becomes more important. What can be seen in Fig. 12 is that the a priori information drives the quality of the retrieval. Several a priori information were tested. The so-called Reference a priori image (i.e. 40-day average of the MODIS retrievals) coud had been used. This was not do that because the Reference is what is used to measure the quality of the data-filling. Furthermore, this information is not always available, and a 40-day averaging a priori might not be a good solution for an instantaneous retrieval (e.g. during a sudden flooding event). It is also interesting to measure the quality of independent 
a priori information (topography, floodability index, etc.) because they can complement the information provided by the observation (MODIS here).

The retrieved surface water maps can have some specificities in terms of uncertainties (i.e. instrument noise, wrong assumptions, limited information, or retrieval errors) or in terms of missing-data (presence of clouds, instrument recalibration, etc.). The methods that are proposed here are not general, they would recommend testing and adjustment for each application. In fact, the a priori information that is proposed (historical record, neighbourhood constrains, elevation, or floodability index) are not specific to one type of observations, but the design of an optimal de-noising or data-filling filter would require to adjust their use to the problem specificities.

\section{Conclusion and perspectives}

In this paper, several determinist filters using several a priori information were introduced to de-noise and fill missing-data in satellite-retrieved surface water maps. It was presented how to exploit these a priori information and how to combine them. The methods presented here are not specific to one type of satellite observations, they are generic and applicable for surface water maps whatever their origin, and flexible enough to be optimised for any particular application. Tests were conducted on SAR- and visible-based retrievals.

Many aspects of the filters described here can be optimised further. For instance:

- A different threshold can be used for the small and large probabilities in GSWO, this would specialise the filters to different environments;

- More complex neighbourhood systems can be expressed, based on large dataset experiments with information on surface type. Adapting the filters to surface water environments would improve their efficiency;

- A pixel-scale neighbourhood statistics could be obtained if a long time record was available. This would ensure that the neighbourhood constrain would be specific for each location, which would be a great improvement, but long time records would be necessary;

- The DEM constraint could use the runoff information in addition to the elevation, this would provide constrains on the flow direction that are more pertinent for local 
inundation patterns;

- The Floodability Index (FI) that was used here could be improved. As mentioned in the text, this FI has been derived using topography information. The model that was used is global and general, and no specific information was provided on the type of environment. An investigation was conducted to know where and when the floodability index is good or not convenient. Based on this study, a more complex FI model could be derived that would be satisfactory in any environment. Furthermore, a newer and more precise topography will be used, this should also improve the FI.

For the missing-data filling, if a historical record is available, a PCA approach could be used (Aires et al., 2014) instead of the adaptative thresholding on the GSWO surface water occurrence that might not be appropriate for all the environments, in particular over densely vegetated areas. By using a same source for the observations and the a priori, the coherency can help accomplishing the data filling.

The most important contribution of this paper is to present several a priori information ressources that can be used to improve satellite water masks. Several ways to exploit this a priori information were presented. Perspectives to improve the filters are numerous, in particular when global and long time records of observations are available. Some parameters of the filters can to be optimised, this could be done for each region or each type of environments.

These sources of a priori information and spatial filters can be used as a post-processing step, for the improvement of satellite surface water maps, but this a priori information could also be used as auxiliary data in retrieval algorithms. In the future, we expect to use another important information for the post-processing of such water maps: the temporal information. This is already an important component of current SAR retrieval algorithms Santoro et al. (2010) and it is expected that this temporal information should leverage the spatial information used in the filters presented here in this paper.

The NASA/CNES Surface Water and Ocean Topography (SWOT) mission, planned for launch in 2021, is specifically designed to provide high-spatial resolution $(\simeq 10 \mathrm{~m})$ and good temporal sampling (22 days repeat) of the extent (and altitude) of continental surface waters thanks to an interferometric Ka-band radar (Rodriguez, 2015; Prigent et al., 2016). Although the SWOT data are expected to deliver a new generation of global water surface extents at 
unprecedented quality and resolution, the availability of this product is still years in the future. Meanwhile, alternative efforts should be pursued to provide the community with the best possible information about the spatial and temporal variations of global surface water extents. Such efforts would also allow for the extension of the SWOT temporal record backward in time, with existing past imagery; this will be a crucial step in assembling multidecadal measurements of surface water variation.

\section{Acknowledgements}

The author would like to thank the French spatial agency CNES ("Centre National d'Etudes Spatiales"), in particular Selma Cherchali for her support, Nicolas Picot for funding a R\&T project in the framework of the SWOT mission (SWOT-SOW-APP-0542-CNE), and Santiago Pena Luque, Roger Fjortoft and Alejandro Bohe for following the progress in this project. Images from the GSWO are courtesy of the European Commission Joint Research Centre, and I would like to thank Jean-François Pekel and colleagues for making them available.

\section{References}

Aires, F., Miolane, L., Prigent, C., Pham, B., Fluet-Chouinard, E., Lehner, B., Papa, F., 2017. A Global Dynamic Long-Term Inundation Extent Dataset at High Spatial Resolution Derived through Downscaling of Satellite Observations. Journal of Hydrometeorology 18, 1305-1325. URL: http://journals.ametsoc.org/doi/10.1175/JHM-D-16-0155.1, doi:10.1175/JHM-D-16-0155.1.

Aires, F., Papa, F., Prigent, C., 2013. A Long-Term, High-Resolution Wetland Dataset over the Amazon Basin, Downscaled from a Multiwavelength Retrieval Using SAR Data. Journal of Hydrometeorology 14, 594-607. URL: http://adsabs.harvard.edu/cgi-bin/

nph-data_query?bibcode=2013JHyMe. . 14 . 594A\&link_type=EJOURNAL, doi:10.1175/ JHM-D-12-093.1.

Aires, F., Papa, F., Prigent, C., Crétaux, J.F., Bergé-Nguyen, M., $2014 . \quad$ Characterization and Space-Time Downscaling of the Inundation Extent over the Inner Niger Delta Using GIEMS and MODIS Data. Journal of Hydrometeorology 15, 

doi $10.1175 /$ JHM-D-13-032.1.

Aires, F., Prigent, C., Fluet-Chouinard, E., Yamazaki, D., Papa, F., Lehner, B., 2018. Comparison of visible and multi-satellite global inundation datasets at high-spatial resolution. Remote Sensing of Environment 216, 427-441. URL: https://doi.org/10.1016/j.rse. 2018.06.015, doi:10.1016/j.rse.2018.06.015.

Bishop, C.M., 1996. Neural networks for pattern recognition. Clarendon Press - Oxford.

Bousquet, P., Ciais, P., Miller, J.B., Dlugokencky, E.J., Hauglustaine, D.A., Prigent, C., Van der Werf, G.R., Peylin, P., Brunke, E.G., Carouge, C., Langenfelds, R.L., Lathière, J., Papa, F., Ramonet, M., Schmidt, M., Steele, L.P., Tyler, S.C., White, J., 2006. Contribution of anthropogenic and natural sources to atmospheric methane variability. Nature 443, 439-443. URL: http://www.nature.com/doifinder/10.1038/nature05132, doi:10.1038/nature05132.

Cao, H., Zhang, H., Wang, C., Zhang, B., 2019. Operational Flood Detection Using Sentinel-1 SAR Data over Large Areas. Water 11, 786-21. URL: https://www.mdpi. com/2073-4441/11/4/786, doi:10.3390/w11040786.

Deledalle, C.A., Denis, L., Tupin, F., Reigber, A., Jager, M., 2014. NL-SAR: A Unified Nonlocal Framework for Resolution-Preserving (Pol)(In)SAR Denoising. IEEE Transactions on Geoscience and Remote Sensing 53, 2021-2038. URL: http://ieeexplore.ieee.org/ document/6905794/, doi:10.1109/TGRS.2014.2352555.

Feng, M., Sexton, J.O., Channan, S., Townshend, J.R., 2014. A global, highresolution (30-m) inland water body dataset for 2000: first results of a topographic-spectral classification algorithm. International Journal of Digital Earth 9, 113-133. URL: http://www.tandfonline.com/doi/full/10.1080/17538947.2015. 1026420, doi:10.1080/17538947.2015.1026420.

Fluet-Chouinard, E., Lehner, B., Rebelo, L.M., Papa, F., Hamilton, S.K., 2015. Development of a global inundation map at high spatial resolution from topographic downscaling of coarse-scale remote sensing data. Remote Sensing of Environment 158, 348-361. 
URL: http://linkinghub.elsevier.com/retrieve/pii/S0034425714004258, doi:10. $1016 / j . r s e .2014 .10 .015$.

Giustarini, L., Hostache, R., Matgen, P., Schumann, G.J.P., Bates, P.D., Mason, D.C., 2013. A Change Detection Approach to Flood Mapping in Urban Areas Using TerraSAR-X. IEEE Transactions on Geoscience and Remote Sensing 51, 2417-2430. URL: http://adsabs.harvard.edu/cgi-bin/nph-data_query?bibcode= 2013ITGRS . .51.2417G\&link_type=EJOURNAL, doi:10 .1109/TGRS . 2012.2210901.

Hornik, K., Stinchcombe, M., White, H., 1989. Multilayer feedforward networks are universal approximators. Neural Networks 2, 359-366.

Lee, J.S., Pottier, E., 2009. Polarimetric radar imaging: from basics to q applications. URL: https://content.taylorfrancis.com/books/download?dac= C2009-0-07032-X\&isbn=9781420054989\&f ormat=googlePreviewPdf.

Lehner, B., Döll, P., 2004. Development and validation of a global database of lakes, reservoirs and wetlands. Journal of Hydrology 296, 1-22. URL: http://adsabs.harvard. edu/cgi-bin/nph-data_query?bibcode=2004JHyd . .296 . . .1L\&link_type=EJOURNAL, doi:10.1016/j.jhydrol.2004.03.028.

Lehner, B., Verdin, K., Jarvis, A., 2008. New global hydrography derived from spaceborne 810 \ elevation data. Eos, Transactions American Geophysical Union 89, 93-94. URL: http: //doi.wiley.com/10.1029/eost2008E010, doi:10.1029/eost2008E010.

Matgen, P., Hostache, R., Schumann, G., pfister, L., hoffmann, L., Savenije, H.H.G., . Towards an automated SAR-based flood monitoring system: Lessons learned from two 1) case studies 36, 241-252. URL: https://www.sciencedirect.com/science/article/ pii/S1474706510002160.

Mueller, N., Lewis, A., Roberts, D., Ring, S., Melrose, R., Sixsmith, J., Lymburner, L., McIntyre, A., Tan, P., Curnow, S., Ip, A., 2016. Water observations from space: Mapping surface water from 25years of Landsat imagery across Australia. Remote Sensing of Environment 174, 341-352. URL: http://dx.doi.org/10.1016/j.rse.2015.11.003, doi: $10.1016 /$ j.rse.2015.11.003. 
Papa, F., Prigent, C., Aires, F., Jimenez, C., Rossow, W.B., Matthews, E., 2010. Interannual variability of surface water extent at the global scale, 1993-2004. Journal of Geophysical Research: Atmospheres (1984-2012) 115. URL: http://onlinelibrary.wiley.com.ezproxy.cul.columbia.edu/doi/10.1029/ 2009JD012674/full, doi:10.1029/2009JD012674.

Pekel, J.F., Cottam, A., Gorelick, N., Belward, A.S., 2016a. High-resolution mapping of global surface water and its long-term changes. Nature 540, \418-422. URL: http://adsabs.harvard.edu/cgi-bin/nph-data_query?bibcode= 2016Natur.540 . 418P\&link_type=EJOURNAL, doi:10.1038/nature20584.

Pekel, J.F., Cottam, A., Gorelick, N., Belward, A.S., 2016b. High-resolution mapping of global surface water and its long-term changes. Nature , 1-16URL: http://www . nature. com/doifinder/10.1038/nature20584, doi:10.1038/nature20584.

Pham-Duc, B., Prigent, C., Aires, F., 2017. Surface Water Monitoring within Cambodia and the Vietnamese Mekong Delta over a Year, with Sentinel-1 SAR Observations. Water 9, 366-21. URL: http://www.mdpi.com/2073-4441/9/6/366, doi:10.3390/w9060366.

Plank, S., Jüssi, M., Martinis, S., Twele, A., 2017. Mapping of flooded vegetation by means of polarimetric Sentinel-1 and ALOS-2/PALSAR-2 imagery 38, 3831-3850. URL: https://www.tandfonline.com/doi/full/10.1080/01431161.2017.1306143, doi:10. $1080 / 01431161.2017 .1306143$

Prigent, C., Lettenmaier, D.P., Aires, F., Papa, F., 2016. Toward a HighResolution Monitoring of Continental Surface Water Extent and Dynamics, at Global Scale: from GIEMS (Global Inundation Extent from Multi-Satellites) to SWOT (Surface Water Ocean Topography). Surveys in Geophysics 37, 339-355. URL: http://adsabs.harvard.edu/cgi-bin/nph-data_query?bibcode=2016SGeo... 37 . 339P\&link_type=EJOURNAL, doi:10.1007/s10712-015-9339-x.

Prigent, C., Matthews, E., Aires, F., Rossow, W.B., 2001. Remote sensing of global wetland dynamics with multiple satellite data sets. Geophysical Research Letters 28, 4631-4634. URL: http://adsabs.harvard.edu/cgi-bin/nph-data_query?bibcode= 2001GeoRL . .28.4631P\&link_type=EJOURNAL, doi:10.1029/2001GL013263. 
Tulbure, M.G., Broich, M., Stehman, S.V., Kommareddy, A., 2016. Surface water extent dynamics from three decades of seasonally continuous Landsat time series at subin land surface water dynamics since the 1990s and relation to population pressure. Geonph-data_query?bibcode=2012GeoRL . 39.8403P\&link_type=EJOURNAL, doi:10.1029/ 2012GL051276.

Prigent, C., Papa, F., Aires, F., Rossow, W.B., Matthews, E., 2007. Global inundation dynamics inferred from multiple satellite observations, 1993-2000. Journal of Geo\ physical Research: Atmospheres (1984-2012) 112. URL: http://onlinelibrary. wiley.com.ezproxy.cul.columbia.edu/doi/10.1029/2006JD007847/full, doi: $10.1029 / 2006$ JD007847

Pulvirenti, L., Chini, M., Pierdicca, N., guerriero, L., Ferrazzoli, P., 2011a. Flood monitoring using multi-temporal COSMO-SkyMed data: Image segmentation and signature interpretation 115, 990-1002. URL: https://www.sciencedirect.com/science/article/pii/ S0034425710003408.

Pulvirenti, L., Pierdicca, N., Chini, M., guerriero, L., 2011b. An algorithm for operational flood mapping from synthetic aperture radar (SAR) data based on the fuzzy logic 11 . URL: https ://www . earth-prints .org/handle/2122/7314.

Rodriguez, E., 2015. Surface Water and Ocean Topography Mission (SWOT). Technical Report JPL D-61923. NASA JPL.

Santoro, M., Wegmüller, U., Askne, J.I.H., 2010. Signatures of ERS-Envisat Interferometric SAR Coherence and Phase of Short Vegetation: An Analysis in the Case of Maize Fields. IEEE Transactions on Geoscience and Remote Sensing 48, 1702-1713. URL: http://adsabs.harvard.edu/cgi-bin/nph-data_query?bibcode= 2010ITGRS . .48.1702S\&link_type=EJOURNAL, doi:10.1109/TGRS . 2009.2034257.

Shen, X., Wang, D., Mao, K., Anagnostou, E., Hong, Y., 2019. Inundation Extent Mapping by Synthetic Aperture Radar: A Review. Remote Sensing 2013, Vol. 5, Pages 687-715 11, 879-17. URL: https://www.mdpi.com/2072-4292/11/7/879, doi:10.3390/rs11070879. 
continental scale in a semi-arid region. Remote Sensing of Environment 178, 142-157.

URL: http://linkinghub.elsevier.com/retrieve/pii/S0034425716300621, doi:10. $1016 /$ j.rse.2016.02.034.

Verpoorter, C., Kutser, T., Seekell, D.A., Tranvik, L.J., 2014. A global inventory of lakes based on high-resolution satellite imagery. Geophys. Res. Lett. 41, 6396-6402. URL: http://doi.wiley.com/10.1002/2014GL060641, doi:10.1002/2014GL060641.

Westerhoff, R.S., Kleuskens, M.P.H., Winsemius, H.C., Huizinga, H.J., Brakenridge, G.R., Bishop, C., 2013. Automated global water mapping based on wide-swath orbital synthetic-aperture radar. Hydrol. Earth Syst. Sci. 17, 651-663. URL: http: //www.hydrol-earth-syst-sci.net/17/651/2013/, doi:10.5194/hess-17-651-2013.

Wilson, A.M., Jetz, W., 2016. Remotely Sensed High-Resolution Global Cloud Dynamics for Predicting Ecosystem and Biodiversity Distributions. PLoS Biol 14, 1-20. URL: http:// dx.plos.org/10.1371/journal.pbio.1002415, doi:10.1371/journal.pbio.1002415.

Winsemius, H., Jongman, B., Veldkamp, T., Hallegatte, S., Bangalore, M., Ward, P., 2015. Disaster risk and poverty: assessing the global exposure of the poor to floods and droughts. EGU General Assembly 2015 17, 3225. URL: http://adsabs.harvard. edu/abs/2015EGUGA . .17.3225W.

Yamazaki, D., Ikeshima, D., Tawatari, R., Yamaguchi, T., O’Loughlin, F., Neal, J.C., Sampson, C.C., Kanae, S., Bates, P.D., 2017. A high-accuracy map of global terrain 口 elevations. Geophysical Research Letters 44, 5844-5853. URL: http://adsabs.harvard. edu/cgi-bin/nph-data_query?bibcode=2017GeoRL . .44.5844Y\&link_type=EJOURNAL, doi:10.1002/2017GL072874.

Yamazaki, D., Trigg, M.A., Ikeshima, D., 2015. Development of a global $\sim 90 \mathrm{~m}$ water body map using multi-temporal Landsat images. Remote Sensing of Environment 171, 337-351. URL: http://linkinghub.elsevier.com/retrieve/pii/S0034425715301656, doi:10. $1016 / j . r s e .2015 .10 .014$. 